\title{
GOAL IMPACT INFLUENCES THE EVALUATIVE COMPONENT OF PERFORMANCE MONITORING: EVIDENCE FROM ERPS
}

Severo, Mario Carlo ${ }^{1}$, Walentowska, Wioleta ${ }^{1,2}$, Moors, Agnes ${ }^{1,3}, \&$ Pourtois, Gilles ${ }^{1}$

\footnotetext{
${ }^{1}$ Cognitive \& Affective Psychophysiology Laboratory, Department of Experimental Clinical and Health Psychology, Ghent University, Belgium

${ }^{2}$ Psychophysiology Laboratory, Institute of Psychology, Jagiellonian University in Krakow, Poland

${ }^{3}$ Research Group of Quantitative Psychology and Individual Differences, Center for Social and Cultural Psychology, KU Leuven, Belgium
}

Running head: Goal impact and ERPs

Corresponding address:

Gilles Pourtois

Department of Experimental Clinical \& Health Psychology

Ghent University

Henri Dunantlaan 2

9000 Ghent

Belgium

email address: Gilles.Pourtois@UGent.be

contact number: +3292649144 


\section{Abstract}

Successful performance monitoring (PM) requires continuous assessment of context and action outcomes. Electrophysiological studies have reliably identified event-related potential (ERP) markers for evaluative feedback processing during PM: the Feedback-Related Negativity (FRN) and $P 3$ components. The functional significance of FRN remains debated in the literature, with recent research suggesting that feedback's goal relevance can account for FRN (amplitude) modulation, apart from its valence or expectedness alone. Extending this account, the present study assessed whether graded differentiations in feedback's relevance or importance to one's goal (referred to as goal impact) would influence PM at the FRN (and P3) level. To this end, we ran a within-subject crossover design experiment in which 40 participants completed two standard cognitive control tasks (Go/No Go and Simon), while 64-channel electroencephalography was recorded. Critically, both tasks entailed similar reward processing but systematically varied in goal impact assignment (high vs. low), manipulated through their supposed diagnosticity for daily life functioning and activation of social comparison. ERP results showed that goal impact reliably modulated FRN in a general manner. Irrespective of feedback valence, it was overall less negative in the high compared to the low impact condition, suggesting a general decrease in feedback monitoring in the former compared to the latter condition. These findings lend support to the idea that PM is best conceived operating not solely based on motor cues, but is shaped by motivational demands.

Keywords: ERP, FRN, P3, Performance monitoring, Goal relevance, Goal impact 


\section{Introduction}

Successful attainment of goal-directed behavior in an ever-changing environment necessitates continuous monitoring of actions and evaluation of decision outcomes. The human cognitive system can rapidly detect mismatches between observed and predicted and/or desired outcomes of the action and carry out corrective measures to make sure that goals are achieved. Performance monitoring $(\mathrm{PM})$ is one among several metacognitive executive functions that is of utmost importance in daily life (Botvinick \& Braver, 2015; Cunillera et al., 2012). It relies on information processing related to internal states or values (i.e., motor-based cues signaling correct or erroneous responses) and/or external events and incentives (i.e., external feedback indicating losses and gains; Ullsperger et al., 2014a, 2014b; Koban \& Pourtois, 2014). PM is flexible in the sense that it can readily switch between these two information cues depending on which one is available at a certain time. In this way, a lack of evidence from one cue (e.g., motor-based cue) can drive dependence on another cue (e.g., external feedback; Stahl, 2010; Bediou et al., 2012; Ullsperger et al., 2014b; Walentowska et al., 2016).

Through the years, a wealth of research has unraveled the behavioral, autonomic, and brain correlates of PM (see Ullsperger et al., 2014a, 2014b; Koban \& Pourtois, 2014, for reviews). In the electrophysiological domain, the Feedback-Related Negativity (FRN) has been consistently linked to the evaluative component of PM. FRN is a negative-going event-related potential (ERP) component elicited after receiving negative (or unexpected) performance feedback, peaking around 250-300 ms after stimulus onset over frontal electrodes along the midline (Miltner et al., 1997). A frontal N2 component elicited by negative feedback usually gives rise to the FRN (Hajihosseini \& Holroyd, 2013; Ullsperger et al., 2014b). It has been suggested that FRN is driven by dopaminergic-dependent reward prediction error signals generated by specific fronto-striatal loops (Holroyd \& Coles, 2002; Frank et al., 2005; Walsh \& Anderson, 2012; Sambrook \& Goslin, 2015). This component is hypothesized to be 
generated mainly in the dorsal compartment of the anterior cingulate cortex by executive control mechanisms dedicated for error detection and cognitive control (Falkenstein et al., 1990; Gehring et al., 1993, 2012; Miltner et al., 1997; Reinhart \& Woodman, 2014).

Ever since, systematic (amplitude) modulations of the FRN due to manipulations of specific emotional or cognitive features of the feedback stimulus have been documented in the literature. For instance, it has been shown that FRN is primarily sensitive to feedback valence, with its amplitude being enhanced by negative compared to positive feedback (Miltner et al., 1997; Nieuwenhuis et al., 2004). Similarly, monetary losses elicit greater FRN amplitude compared to gains (Gehring \& Willoughby, 2002). In addition, feedback probability and hence expectedness modulates FRN, with unexpected events usually generating larger FRN amplitude than expected ones (Hajcak et al., 2007; Pfabigan et al., 2011; von Borries et al., 2013; Ferdinand et al., 2012). Finally, feedback presentation modes have been demonstrated to influence the morphology of the FRN, with a blocked feedback type presentation yielding more distinct peaks compared to a randomized type presentation for example (Pfabigan et al., 2014).

The P3 is another evaluative ERP component of PM commonly investigated and reported alongside the FRN (Bellebaum \& Daum, 2008; Hajcak et al., 2005; Luu et al., 2009; Nieuwenhuis et al., 2005; Pfabigan et al., 2011; San Martin, 2012; Wu \& Zhou, 2009; Yeung et al., 2005; Yeung \& Sanfey, 2004). It is a positive-going component that peaks around 300$600 \mathrm{~ms}$ after feedback onset at the centroparietal recording sites (Desmedt et al., 1965; Sutton et al., 1965). The P3 component likely reflects the motivational significance of the feedback stimulus (Yeung \& Sanfey, 2004; San Martin, 2012). Based on the independent coding model (Yeung \& Sanfey, 2004), the P3 component is sensitive to the magnitude of the value of the feedback. This is supported by subsequent studies revealing that this long latency component tends to exhibit larger peak for outcomes with a larger value than ones with a smaller value 
(Bellebaum et al., 2010; Goyer et al., 2008; Gu et al., 2011; Kreussel et al., 2012; Polezzi et al., 2010; San Martin, 2012; Toyomaki and Murohashi, 2005; Wu \& Zhou, 2009).

From the current literature, however, a lack of consensus remains as to which dimension of the feedback stimulus critically drives the FRN (amplitude) variations (Hajihosseini \& Holroyd, 2013; Proudfit, 2015; Ullsperger et al., 2014b). While some authors suggest that the FRN mainly codes for valence irrespective of expectedness (Hajcak et al., 2006; Nieuwenhuis et al., 2004), others propose the exact opposite view that the FRN codes for expectedness independent of valence (Ferdinand et al., 2012). Adding to this literature, we have recently conducted studies suggesting that the relevance of the feedback stimulus to an individual's goal is an additional factor that influences PM, including the FRN (Walentowska et al., 2016). In this earlier work, goal relevance was broken down into three partly dissociable components: (1) task relevance, which refers to the degree to which a (feedback) stimulus signals that a goal can be implemented, (2) goal informativeness or reliability, which refers to the degree to which a (feedback) stimulus is informative about the satisfaction status of a goal, and (3) goal impact, which refers to the degree of goal satisfaction or dissatisfaction that is signaled by a (feedback) stimulus. Walentowska et al. (2016) examined the influence of goal relevance in the sense of goal informativeness (i.e., the second meaning of goal relevance) on the FRN component. Critically, these results showed that the FRN differentiating negative from positive feedback was only elicited when the feedback was deemed goal relevant (as opposed to being goal irrelevant). Moreover, in case the feedback was viewed as goal irrelevant by the participant, a switch towards enhanced internal monitoring (at the level of the response-locked ERN component) was observed. As such, these previous results confirmed that PM is best conceived of as a flexible process that is able to switch from using external to internal cues depending on the goal relevance of the feedback stimulus. Furthermore, we proposed that a hierarchical model of PM (but see also Holroyd \& Yeung, 2012) could account for these results, with goal 
relevance likely operating at the superordinate level, followed by expectedness at the intermediate level, and valence at the subordinate level.

The aim of the present study was to extend this line of research by investigating the influence of goal relevance in the sense of goal impact (i.e., the third meaning of goal relevance) on the FRN. In addition, while the previous studies compared the presence with the absence of goal relevance, in the present study we compared two conditions that were both goal relevant but they differed in their perceived degree of relevance or importance. In everyday life, more often than not, we deal with information that is goal relevant to a greater or lesser extent. The present study was set out to examine whether differences in the degree of goal relevance could have a graded influence on PM. To illustrate, picture yourself giving a presentation of a new study proposal in front of colleagues and noticing the puzzled look on their faces as you describe the methods. Now, imagine giving the same presentation, but this time, for a selection committee for grant applications and receiving exactly the same reaction. While the feedback (i.e., the confused look of the audience) is relevant to the goal of giving a clear and wellorganized presentation in both scenarios, it probably carries a larger weight in the latter case. In the same way, marks on exams carry a greater degree of importance to the goal of performing well in a course compared to marks on practice modules. These are a few real-life situations in which feedback may carry a graded level of goal relevance. For purposes of simplicity and consistency, we refer to the definition of goal relevance as goal impact from now on.

We investigated whether feedback that varies in its goal impact influences PM at the level of the FRN and P3 components. To this end, we designed a study with two conditions that differed in the goal impact of the feedback (high vs. low), while keeping reward probability equal in these two conditions. Similar to the studies of Walentowska et al. (2016), participants were more likely to receive negative than positive feedback in both goal impact conditions. The experiment used a within-subject crossover design in which a large number of participants 
completed two experimental tasks that differed in their goal impact, with an interchangeable assignment of each goal impact (either high or low) to each task across them. Sixty-four channel electroencephalography (EEG) was recorded concurrently to measure possible amplitude variations of the FRN (and subsequent P3) component depending on goal impact. Two speeded versions of standard cognitive control tasks were employed in which participants had to respond as fast and as accurately as possible before an arbitrary response deadline. After each response, participants were provided with performance feedback. Since reaction times (RTs) were inherently variable across time and conditions, participants had to thoroughly monitor the feedback in order to assess their actions as goal conducive (i.e., correct and fast enough) or not (i.e., correct but too slow, or incorrect). To ensure that feedback expectedness was constant, a stringent trial-by-trial calibration of the response deadline was applied, allowing for asymmetry in reward probability (i.e., frequent negative feedback vs. deviant positive feedback). More importantly, to manipulate the goal impact of the feedback, these tasks were interchangeably assigned to either high or low impact conditions through the use of a cover story. Participants were misled to believe that both tasks were self-regulation measures but differed in their diagnosticity and the presence of social comparison. In the high impact condition, participants were told that the task was diagnostic of good academic performance and successful interpersonal relationships and that their overall performance would be compared to the scores of a peer group. In the low impact condition, participants did not receive information on the diagnosticity of the task and their performance was not compared with that of their peers. We assumed that this manipulation would lead to differences in the impact of the performance feedback for the goals of maintaining one's social status or self-esteem. These are goals that are generally considered to be important for the majority of people (Rosenberg et al., 1995; Baumeister et al., 2003). 
In line with our previous work (Walentowska et al., 2016), we predicted a significant interaction effect between feedback valence (positive vs. negative) and goal impact (high vs. low). More specifically, we reckoned that the FRN reflecting greater relative negativity for negative than positive feedback would be more pronounced in the high impact condition than in the low impact condition. This valence-related modulation in the FRN would be explained by the differential impact of feedback on the goal of maintaining social status or self-esteem, and hence the differential need to carefully monitor performance. We expected a similar effect to be captured by the P3 component. Additionally, to control for the possible influence of the goal impact manipulation on the arousal level of participants, we collected subjective arousal ratings, as well as electrocardiography (ECG) for heart rate variability (HRV) analysis as a more objective physiological marker.

Even though our main prediction and focus was on possible changes in externally-driven PM (at the FRN level) as a function of goal impact, we additionally looked into its possible effect on internal monitoring (at the response-locked ERPs level), in line with our previous work (see Walentowska et al., 2016). Internal or motor-based PM is reflected in the ErrorRelated Negativity (ERN) and the Correct-Related Negativity (CRN) components. The ERN is a negative deflection elicited shortly after error commission (Falkenstein et al., 1990), while the $\mathrm{CRN}$ is a similar negative-going component generated after correct responses, albeit smaller in amplitude (Allain et al., 2004). For the sake of completeness, as well as direct comparison with earlier ERP studies (e.g. Holroyd \& Coles, 2002; Stahl, 2010; Walentowska et al., 2016), we also report the results of our auxiliary analyses on these response-locked ERP components.

\section{Materials and Methods}

\subsection{Participants}

Forty-four university students participated in the experiment in exchange for $€ 30$ 
compensation $^{1}$. Four participants had to be removed due to excessive noise and artifacts during the EEG recording, keeping a total of 40 (15 males; aged $=20-29$ years old; MEAN $=22.88$, $\mathrm{SD}=2.56)$ to be included in the analyses. All participants were right-handed and had normal or corrected-to-normal vision. They gave written informed consent to take part in the study in accordance to the Declaration of Helsinki. The study was approved by the local ethics committee of Ghent University.

\subsection{Experimental paradigm, design, and tasks}

Central to the manipulation of the degree of goal impact (high vs. low) in a within-subject design was the use of two tasks that could be assigned interchangeably to both goal impact conditions, but at the same time, would yield comparable reward probabilities. Therefore, we devised a crossover experimental design utilizing speeded versions of two standard cognitive control tasks: (1) an extensively validated Go/No Go Task (see Aarts \& Pourtois, 2010, 2012; Koban et al., 2012; Pourtois, 2011; Vocat et al., 2008; Walentowska et al., 2016) and (2) a Simon Task (Simon \& Ruddel, 1967). In both tasks, participants had to respond as fast and as accurately as possible before an arbitrary response deadline in order to receive positive feedback on task performance. Because of the variable nature of RTs, participants had to monitor the performance feedback to evaluate the goal conduciveness of their actions (i.e. whether they achieved a correct and fast response). Figure 1 presents a schema of the experimental design.

\footnotetext{
${ }^{1}$ We aimed for at least 20 subjects per each group of the between-subjects factor of sequence (see here below) to make sure we had sufficient power (see Simmons et al., 2011).
} 


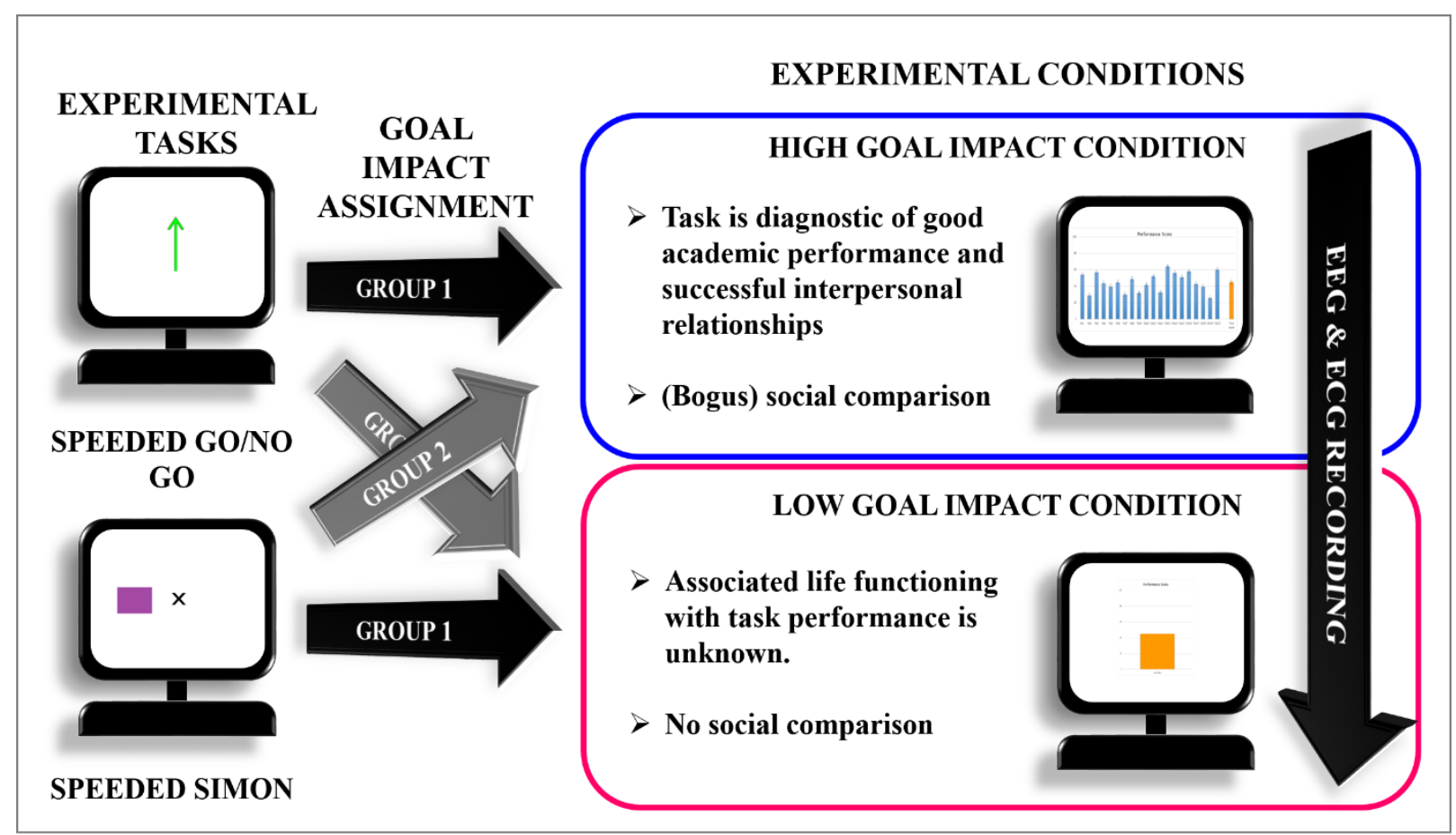

Figure 1. Schema of the experimental design. A within-subject crossover design experiment was devised whereby two speeded versions of standard cognitive control tasks (Go/No Go \& Simon) were interchangeably assigned to two goal impact conditions (high vs. low) while continuous EEG and ECG were recorded. Using an elaborate cover story, participants were misled to believe the differences in the motivational significance of performance feedback in the two tasks. In the high impact condition, they were told that the task was diagnostic of good academic standing and successful interpersonal relationships and their performance was compared to a peer group. In the low impact condition, important life functioning or ability associated with the task was not provided and their performance was not compared. The assignment of goal impact and the order of presentation of the two tasks were fully counterbalanced across all participants.

A cover story was used to effectively manipulate the degree of goal impact. The two tasks were presented as self-regulation measures and were assigned to either high or low impact conditions. In the high impact condition, participants were misled to believe that the task had been established by previous research to be diagnostic of good academic performance and successful interpersonal relationships. Moreover, they were told that an earlier study of the experimenters found a strong correlation between the task performance of 20 previously tested participants and their university course grades. A bar graph containing the alleged performance scores (i.e., combined speed and accuracy scores with a maximum of 100 points that were used as performance benchmark) of these students was shown during instructions. To further 
encourage social comparison as a way to strengthen the enhanced goal impact of this condition, participants were told that their performance score would be evaluated in conjunction to this reference peer group and performance benchmark. At the end of each block of the task, participants received a performance score for the respective block. After the final block, they received their overall performance score, averaged across blocks, together with the bogus performance of their peers (i.e., the same bar graph that was presented during the instructions).

In the low impact condition, participants were not provided with information on the diagnostic properties of the task for their future life functioning. They were informed that the task was still in the stage of piloting and validation, and hence, that their performance could not be compared yet to previously tested subjects. However, they were told that they would still receive an evaluation of their overall performance (i.e., their performance score) and that this score would be used as a benchmark for future experiments. At the end of each block, they received a performance score for the respective block. After the final block, they were shown a bar graph representing their own bogus performance score.

It could be noted that the two experimental tasks tap into slightly different executive functions, with the Go/No Go Task being a motor-inhibition task and the Simon Task being a spatial-discrimination task (with interference), but we reckoned that this difference would facilitate the interchangeable tagging of goal impact conditions across participants and would bolster the credibility of the cover story. Furthermore, because of the crossover experimental design implemented here, we did not need to consider the differences in task characteristics as a factor in the analyses. Both tasks were presented using E-prime software (V2.0., Psychology Software Tools Inc., Sharpsburg, PA). All visual stimuli related to each task were presented on a 21-inch CRT screen. A detailed description of both tasks follows. 


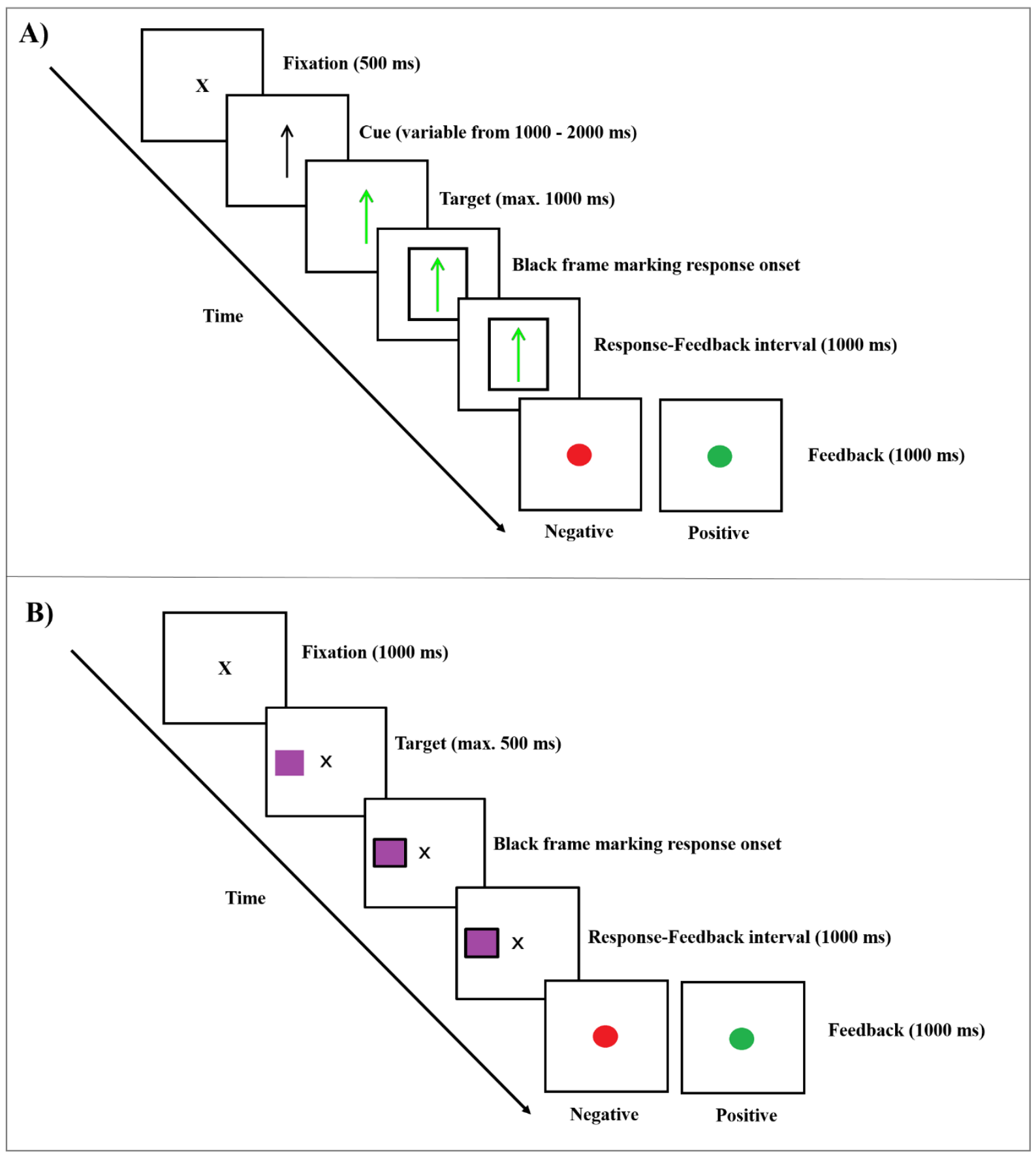

Figure 2. Task trial sequence. (A) Speeded Go/No Go Task (here illustrated for Go trials, followed by hits; see Methods): Each trial started with a centrally presented fixation cross on a white background for $500 \mathrm{~ms}$. Next, a black arrow serving as cue appeared with a 1000-2000 ms jittered duration. Following this was the presentation of the target for a maximum duration of $1000 \mathrm{~ms}$. Participants were instructed to press a predefined key from the response box as fast as possible. A black frame appeared around the target consequent to motor response and stayed on screen for $1000 \mathrm{~ms}$ until feedback onset, serving as response-feedback interval. Fast hits were always followed by a positive feedback (a green dot), while slow hits were always followed by a negative feedback (a red dot). (B) Simon Task: Each trial started with a centrally presented fixation cross on a white background for 1000 ms. Then, a color-coded square appeared on the side of the screen as target for a maximum duration of $500 \mathrm{~ms}$. Participants were asked to press predefined keys from the response box as fast as possible depending on the 
stimulus-response mapping condition assigned to them. A black frame was displayed around the target upon motor response and remained on screen for $1000 \mathrm{~ms}$ until feedback onset, serving as response-feedback interval. Similar to the other task, fast hits were always followed by a positive feedback (a green dot), while slow hits were always followed by a negative feedback (a red dot).

\subsubsection{Go/No Go Task}

Figure 2A depicts a sample trial sequence for the task. Each trial started with a black fixation cross lasting for $500 \mathrm{~ms}$. Next, a black arrow ('cue') was presented, either oriented up or down, for a variable duration of 1000 to $2000 \mathrm{~ms}$. Then, the black arrow became either green or turquoise, while its orientation either remained identical or shifted in the opposite (in-plane) orientation, appearing at a maximum duration of 1000 ms. Critically, this (second) event defined the trial type. A green arrow with an unchanged orientation ('target') required participants to quickly press a predefined key on the response box with the index finger of the right hand ('Go trials'). A green arrow with a flipped orientation or a turquoise arrow with an unchanged orientation (both 'non-targets') necessitated withholding of a response ('No Go trials'). All cues, targets, and non-targets were arrows presented in the center of the screen on a white background. A black frame appeared around the target consequent to motor responses (either correct: 'hits' or incorrect: 'false alarms'). This event lasted for $1000 \mathrm{~ms}$, serving as a responsefeedback interval. In the absence of a motor response, targets and non-targets without a black frame remained on the screen for $1000 \mathrm{~ms}$. Finally, a color-coded symbolic feedback cue was displayed for $1000 \mathrm{~ms}$ subsequent to the motor response type. Participants saw either a red or green dot in the center of the screen on a white background. They got a green dot, indicating a positive feedback, for a correct and fast response to Go trials ('fast hit') and successful withholding of a response to No Go trials ('correct inhibition'). Conversely, they received a red dot, signifying a negative 
feedback, for a correct but slow response ('slow hit') or the lack of a response ('omission') to Go trials or non-inhibition of response ('false alarm') to No Go trials. Participants had to complete three blocks of this task. A block was composed of 56 trials of which 40 were Go trials and 16 were No Go trials.

Unbeknownst to participants, an online adaptive algorithm was implemented to determine a strict response deadline procedure in Go trials, setting the RT limit to 300 ms at the beginning of the experiment (based on Vocat et al., 2008; Walentowska et al., 2016). This limit was then adjusted trial by trial as a function of the immediate preceding trial history, that is, the average of the current and previous RTs. This calibration allowed for stringent delineation of fast and slow hits and generation of a smaller percentage of the former (1/3) than the latter (approximately 2/3), thereby making positive feedback less expected relative to negative feedback. The advantages for the use of this algorithm are twofold. First, participants had to rely on the external feedback stimulus presented to them on each trial to infer whether their responses (during Go trials) were goal conducive (i.e., fast hits) or not (i.e., slow hits). The high uncertainty in deciphering variable RTs should motivate participants to rely on the feedback for behavioral adjustments and to evaluate this feedback as highly informative of their performance. Second, because fast hits (corresponding to rewarded events) were fairly challenging to attain (Aarts \& Pourtois, 2010, 2012; Dhar \& Pourtois, 2011; Dhar et al., 2011; Koban et al., 2010, 2012; Vocat et al., 2008; Walentowska et al., 2016), participants should be highly engaged in the task (Atkinson \& Feather, 1966), thereby avoiding habituation or fatigue (Walentowska et al., 2016).

\subsubsection{Simon Task}

Figure $2 \mathrm{~B}$ depicts a sample trial sequence for the task. Each trial started with a black 
fixation cross on a white background, lasting for $1000 \mathrm{~ms}$. Then, a color-coded square ('target') appeared for a maximum duration of $500 \mathrm{~ms}$. The target, either yellow or violet, appeared on either the left or right side of the fixation cross. Participants had to press either a right-side key with the index finger of their right hand or a left-side key with the index finger of their left hand, depending on the assigned stimulus-response mapping. For instance, they had to press the right-side key for a yellow square and the left-side key for a violet square (or vice-versa). This stimulus-response mapping was counterbalanced across all participants. Crucially, the compatibility between the stimulus location and the response location defined the trial type. On compatible trials, participants had to press the right key to squares presented on the right, or the left key to squares presented on the left. On incompatible trials, they had to press the right key to squares presented to the left, or the left key to squares presented on the right. A black frame was displayed around the target after a (correct or incorrect) response was made. This event lasted for $1000 \mathrm{~ms}$, serving as a response-feedback interval. If no response was made, the target without a black frame remained on the screen for 1000 ms. Finally, a feedback cue was presented in the center of the screen on a white background for 1000 ms subsequent to the motor response type. A green dot indicating a positive feedback was given after responses that were both correct and fast ('fast hits'). A red dot signifying a negative feedback was provided after correct but slow responses ('slow hits'), incorrect responses ('errors'), or when no response was made ('omission'). Participants had to complete three blocks of this task. A block was composed of 56 trials of which 38 were compatible trials and 16 were incompatible trials. The unequal proportion of trial types was intended to increase the likelihood to obtain a Simon effect (see Simon \& Ruddel, 1967). 
Similar to the Go/No Go Task, an online adaptive algorithm was implemented to define a strict response deadline procedure, setting the RT limit to $350 \mathrm{~ms}$ at the beginning of the experiment based on extensive piloting. As described earlier in the previous task, this limit was adjusted trial by trial based on the average of the current and previous RTs. It delineated fast from slow hits, generating a smaller percentage of the former relative to the latter, thereby, making positive feedback less expected than negative feedback.

\subsubsection{Manipulation Check}

At the end of each task, participants were asked a series of questions to test if our manipulations of goal impact were successful. They were asked to rate for each task using a Visual Analog Scale (VAS) ranging from 0 (not at all) to 100 (a lot) to what extent they

(a) disliked making an error on the task, (b) liked making a correct response on the task, (c) disliked receiving negative feedback on the task, (d) liked receiving positive feedback on the task, and (e) how easy the task was for them knowing that their mean performance score was compared to that of others in the high impact condition, and how easy the task was for them knowing that their mean performance score was not compared to that of others in the low impact condition. After this, they were also asked to rate how aroused they were while completing the task using the Self-Assessment Manikin (SAM; Bradley \& Lang, 1994).

\subsection{Experimental procedure}

After having signed the informed consent, the experimenter attached the ECG and EEG sensors. Participants were tested in a dimly lit, sound-attenuated, and electrically shielded cabin, while seating at a distance of $\sim 80 \mathrm{~cm}$ from the computer monitor. Prior to the start of the 
testing session, they were given information on the difference in goal impact of the two experimental tasks. This was followed by a five-minute resting-state ECG recording, which served as the baseline recording of participants' arousal level.

The test session was composed of two parts, each corresponding to one of the two taskimpact assignments. The order of presentation of the experimental tasks (Go/No Go vs. Simon) and impact condition (high vs. low) were fully counterbalanced across participants. In each part, participants received specific instructions on the task procedure and the goal impact. Next, they performed 32 practice trials and completed the three blocks of the task, which were closed by a presentation of their overall performance scores. Finally, they completed the manipulation checks and subjective arousal ratings. Each part was followed by a five-minute resting-state ECG recording, which served as the post-task recording for the previous task.

After the ECG/EEG equipment had been removed, Dutch versions of three dispositional measures were administered using E-prime software: the Behavioral Avoidance/Inhibition Scale (BIS/BAS; Carver \& White, 1994; Franken, 2002), the Liebowitz Social Anxiety Scale (LSAS; Liebowitz, 1987; van Vliet, 1999), and the Rosenberg Self-Esteem Scale (RSES; Rosenberg, 1965; Franck et al., 2006). A debriefing letter was sent to the participants after all had been tested.

\subsection{Data recording}

Continuous ECG and EEG were recorded at a sampling rate of 512-Hz using $\mathrm{Ag}-\mathrm{AgCl}$ (silversilver chloride) electrodes with a 64-channel ActiveTwo system (BioSemi B. V., Amsterdam, the Netherlands) and were referenced online to the Common Mode Sense (CMS)-Driven Right Leg (DRL) ground. Two ECG electrodes were attached on the left side of the chest cavity, one just below the right clavicle and the other on the lower torso. Sixty-four EEG electrodes were mounted in an elastic cap in accordance to the extended International 10-20 EEG system. Six 
auxiliary electrodes were additionally attached. The horizontal and vertical electrooculography (EOG) signals were monitored by four of these electrodes, positioned above and below the left eye and on each of the outer canthi of the eyes. The remaining two electrodes were placed on the left and right mastoids.

\subsection{Data reduction}

\subsubsection{ECG}

The raw ECG signal was exported to Brain Vision Analyzer 2.0 (Brain Products, GmbH, Munich, Germany), where the difference between the two electrodes was obtained by applying a standard subtraction. The data were then segmented to the following epochs of interest: (a) baseline prior to the tasks, (b) individual blocks of each of the experimental tasks, and (c) post-task recording. Each of these segments were subjected to a heart rate variability (HRV) analysis. HRV reflects the small beat-to-beat differences in the heart rate (or inter-beat intervals, IBI) as a result of the dynamic control of the sympathetic and parasympathetic branches of the autonomic nervous system (Task Force, 1996; Thayer et al., 2009; Lane et al., 2009). Since the heart is dually innervated by these two branches, relative sympathetic activity elevations are associated with increased heart rate, while relative parasympathetic activity increases are associated with decreased heart rate. Thus, the former leads to shorter IBIs, whereas the latter results in longer IBIs (Lane et al., 2009). Offline HRV analysis was implemented using ARTiiFACT software (Kaufmann et al., 2011), following a standard data transformation sequence: (a) an automated R-peak detection, (b) IBI extraction, and (c) artifact detection and correction via cubic spline interpolation of neighboring IBIs. We analyzed the root mean square of successive differences (RMSSD), the most frequently employed HRV parameter in the time domain (Task Force, 1996). This parameter was $\log$-transformed (log10) to achieve normal 
distribution prior to analysis (Laborde et al., 2017). A decrease in RMSSD (relative to the baseline) indicates a higher arousal level, while an increase in RMSSD (relative to the baseline) signifies a lower arousal level.

\subsubsection{EEG}

Offline analysis of the EEG time series was performed using Brain Vision Analyzer 2.0, following a standard data transformation sequence (Keil et al., 2014) with the following steps: (a) 50-Hz notch filtering, (b) re-referencing via linked-mastoid, (c) -500/+1000 ms segmentation around the onset of the feedback stimulus (for FRN and P3), and -500/+500 ms segmentation around the response onset (for ERN/CRN), (d) pre-stimulus baseline correction (from -500 ms to feedback onset) and pre-response baseline correction (from 500 to $-300 \mathrm{~ms}$ prior to the response onset), (e) vertical ocular correction for blink (Gratton et al., 1983), (f) semi-automatic artifact rejection by applying a fixed criterion of $\pm 80 \mu \mathrm{V}$, (g) averaging of the feedback-locked ERPs per each feedback type and impact condition, as well as averaging of the response-locked ERP per each response type and impact condition, and (h) 30-Hz low-pass digital filtering of the ERPs. A priori selection of time windows and electrode sites for the quantification of the ERP components analyzed were mainly based on a previous study (Walentowska et al., 2016). Moreover, as described below, two different (and standard) scoring methods (i.e., peak-to-peak vs. mean amplitude) were implemented, which allowed for comparison of both time window-dependent and relatively independent approaches (Luck, 2014; Luck \& Gaspelin, 2017).

The feedback-locked ERPs were mainly composed of the positive feedback following fast hits and negative feedback following slow hits in the two impact conditions. Feedback consequent to other response types (i.e., correct inhibitions and false alarms in the Go/No Go Task and incorrect responses in the Simon Task) were not analyzed as they were 
not informative for participants, and hence not associated with systematic post-feedback onset ERP effects. In such cases, individuals can evaluate accuracy of their actions by relying on internal monitoring, thus, there was no need to attend to external feedback (see Koban et al., 2012).

We primarily focused on the Feedback-Related Negativity (FRN), which have been associated with the processing of external feedback in earlier ERP studies on PM, alongside the P3 component (Aarts \& Pourtois, 2012; Bismark et al., 2013; Ferdinand et al., 2012; Fischer \& Ullsperger, 2013; Pfabigan et al., 2011, 2014; von Borries et al., 2013; Walsh \& Anderson, 2012; Walentowska et al., 2016). Ways of quantifying FRN typically differ across studies, with some researchers favoring peak-to-peak analysis (e.g. Mushtaq et al., 2016; Ferdinand et al., 2012; Oliveira et al., 2007; Osinsky, 2012; Schaefer et al., 2016), and others favoring the traditional mean amplitude analysis. We employed both methods. For the mean amplitude measure, we took the mean voltage within $250-300 \mathrm{~ms}$ after feedback onset over the $\mathrm{Fz}$ and $\mathrm{FCz}$ channels, as it has been observed that the FRN amplitude is usually maximum at frontocentral electrode positions (Ullsperger et al., 2014a). Alternatively, the FRN amplitude was also determined relative to its preceding peak, namely the P2 or P170 component (i.e., peak-to-peak analysis). In accordance with the existing literature using this method (Mushtaq et al., 2016; Oliveira et al., 2007; Osinsky, 2012; Schaefer et al., 2016), we identified the mean voltage of the P2 component within 180-220 ms over the same electrode positions and subtracted this from the mean voltage of the FRN. As for the P3 component, we simply quantified its amplitude by taking the mean voltage within 300-400 ms over $\mathrm{Pz}$ and POz channels.

For the auxiliary analyses, the response-locked ERPs consisted of motor responses categorized as 'errors' (i.e., false alarms in Go/No Go Task and incorrect responses in Simon Task) and 'hits' (i.e., collapsing fast and slow hits in both tasks). We looked at the ERN and 
the CRN, two ERP components that have been associated with the processing of incorrect and correct responses, respectively (Falkenstein et al., 1990; Allain et al., 2004). The amplitude of the ERN and CRN components were quantified by taking the mean voltage within an interval from $10 \mathrm{~ms}$ prior to until $50 \mathrm{~ms}$ after the motor response over the $\mathrm{Fz}$ and FCz electrode sites.

\subsection{Statistical analyses}

All statistical analyses were carried out with JASP 0.7.0.5.6 (JASP Team, 2016). We employed mixed model ANOVAs that included one between-subjects factor and several within-subject factors to analyze the behavioral and electrophysiological data. For the behavioral data, the ANOVA included the within-subject factors RESPONSE (fast hit vs. slow hit; post-correct vs. post-error) and IMPACT (high vs. low). For the HRV, the ANOVA included the within-subject factors PHASE, in which the recording took place (baseline vs. task vs. post-task), and IMPACT. For the feedback-locked ERP components, the ANOVA included the within-subject factors ELECTRODE (Fz vs. FCz for the FRN; Pz vs. POz for the P3 component),VALENCE (positive vs. negative), and IMPACT, as well as the between-subjects factor SEQUENCE (i.e., the presentation order of the impact conditions: first high then low vs. first low then high). Similarly for the response-locked ERP components, the ANOVA included the within-subject factors ELECTRODE (Fz vs. FCz), RESPONSE (errors vs. hits), and IMPACT. Results were evaluated against an alpha of 0.05. A Greenhouse-Geisser correction was applied when the assumption of sphericity was violated. In such cases, uncorrected degrees of freedom and epsilon values $(\varepsilon)$ are indicated. Significant main or interaction effects are reported first, followed by post-hoc paired $t$ tests when applicable.

\section{Results}




\subsection{Dispositional measure scores}

The scores and standard deviations of the subscales of the dispositional measures (i.e., BIS/BAS, LSAS, and RSES) are summarized in Table 1.

\begin{tabular}{|c|c|c|c|c|}
\hline \multicolumn{5}{|c|}{ DISPOSITIONAL MEASURE SCORES } \\
\hline QUESTIONNAIRES & SUBSCALES & MEAN (SD) & MIN SCORE & MAX SCORE \\
\hline \multirow{4}{*}{$\begin{array}{l}\text { BEHAVIORAL } \\
\text { AVOIDANCE/INHIBITION } \\
\text { SCALES (BIS/BAS) }\end{array}$} & BAS - DRIVE & $12.2( \pm 2.05)$ & 4 & 16 \\
\hline & BAS - FUN-SEEKING & $12.5( \pm 2.20)$ & 4 & 16 \\
\hline & $\begin{array}{l}\text { BAS - REWARD } \\
\text { RESPONSIVENESS }\end{array}$ & $15.9( \pm 2.38)$ & 5 & 20 \\
\hline & BIS & $19.7( \pm 3.28)$ & 7 & 28 \\
\hline \multirow{6}{*}{$\begin{array}{l}\text { LIEBOWITZ SOCIAL ANXIETY } \\
\text { SCALE }\end{array}$} & FEAR & & & \\
\hline & PERFORMANCE & $11.1( \pm 4.66)$ & 0 & 36 \\
\hline & SOCIAL INTERACTION & $10.17( \pm 5.13)$ & 0 & 33 \\
\hline & AVOIDANCE & & & \\
\hline & PERFORMANCE & $8.85( \pm 4.46)$ & 0 & 36 \\
\hline & SOCIAL INTERACTION & $9.43( \pm 5.05)$ & 0 & 33 \\
\hline $\begin{array}{l}\text { ROSENBERG SELF-ESTEEM } \\
\text { SCALE }\end{array}$ & SELF-ESTEEM & $20.43( \pm 4.67)$ & 0 & 30 \\
\hline
\end{tabular}

Table 1. Results of questionnaires.

\subsection{Manipulation check}

The VAS ratings of participants on the manipulation check (see Figure 3) showed no significant differences in disliking making an error, $t_{(39)}=0.397, p=0.694, d=0.063$, liking correct responses, $t_{(39)}=0.651, p=0.519, d=0.103$, disliking negative feedback, $t_{(39)}=0.132, p=$ $0.895, d=0.021$, and liking positive feedback, $t_{(39)}=1.911, p=0.063, d=0.302$, between the high and low impact conditions. However, participants did report a significant difference in how easy they thought the task was considering the presence vs. absence of social comparison in the two tasks, $t_{(39)}=-2.541, p=0.015, d=-0.402$ : They perceived the task in the low impact 
condition, in which the social comparison was absent, to be easier to complete $(M=55.52$, SEM $=2.933)$ than the task in the high impact condition, in which social comparison was present $(M$ $=45.67, S E M=3.105)$.

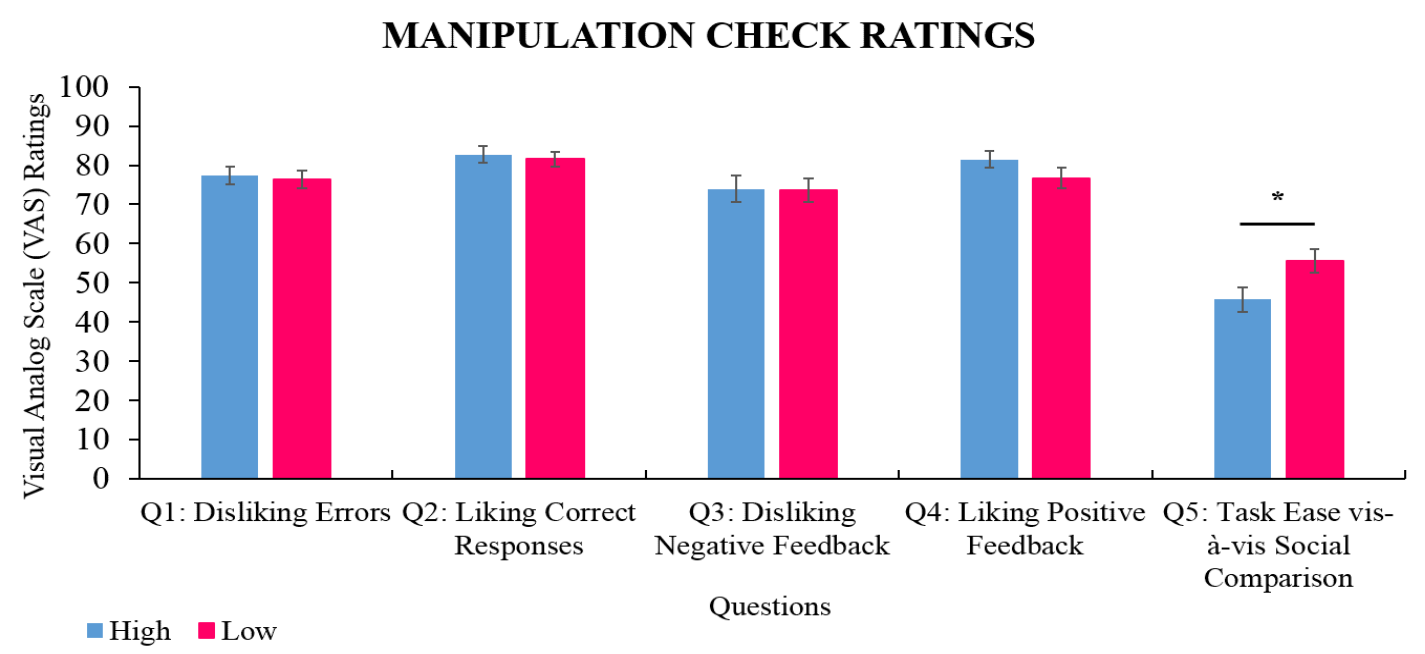

Figure 3. Manipulation check. The VAS ratings (means \pm 1 S.E.M) for questions relating to disliking committing error (Q1), liking making correct responses (Q2), disliking negative feedback (Q3), liking positive feedback (Q4), and task intrusion of the social comparison (Q5) between the two goal impact conditions (high vs. low). Participants judged the task in the low impact condition, in which the social comparison was absent, to be easier to complete than the in the high impact condition, in which social comparison was present. ${ }^{*} p<.05$.

\subsection{Arousal measures: HRV and SAM}

The HRV, as indexed by the log-transformed values of the RMSSD, significantly changed during the baseline recording, the task block recording, and the post-task recording, as evident in the significant main effect of PHASE, $F_{(2,78)}=7.218, p=0.003, \varepsilon=0.825, \eta p^{2}=0.156$. For both impact conditions, there was an increase in RMSSD going from the baseline recording phase $(M=1.58, S E M=0.034)$ to the task blocks recording phase $\left(M_{\text {high impact }}=1.64, S E M=\right.$ 0.029; $\left.M_{\text {low impact }}=1.65, S E M=0.030\right)$, suggesting a decrease in the arousal level. The RMSSD decreased again during the post-task recording $\left(M_{\text {high impact }}=1.62, S E M=0.028 ; M_{\text {low impact }}=\right.$ 
1.63, SEM = 0.030), indicating an increase in the arousal level. There was no significant main effect of IMPACT, $F_{(1,39)}=1.202, p=0.280, \eta p^{2}=0.030$. Also, there was no significant IMPACT x PHASE interaction, $F_{(2,78)}=0.322, p=0.660, \varepsilon=0.738, \eta p^{2}=0.008$, indicating that the impact conditions did not modulate the arousal level of the participants. Corroborating this non-modulatory effect, the SAM arousal ratings showed no significant difference between the two impact conditions, $t_{(39)}=1.662, p=0.104, d=0.263$.

\subsection{Behavioral results}

Results of the different behavioral indices (i.e., accuracy percentages and RTs, error percentages and RTs, and post-error slowing; see Table 2 for a summary) suggest that the actual performances of the participants were balanced between the two impact conditions for both experimental tasks.

\begin{tabular}{|c|c|c|c|c|c|c|}
\hline \multicolumn{7}{|c|}{ PERFORMANCE MONITORING BEHAVIORAL INDICES } \\
\hline & & & \multicolumn{4}{|c|}{ IMPACT } \\
\hline & & & \multicolumn{2}{|c|}{ HIGH } & \multicolumn{2}{|c|}{ LOW } \\
\hline & & & ACCURACY (\%) & $\begin{array}{l}\text { REACTION TIME } \\
\text { (ms) }\end{array}$ & ACCURACY (\%) & $\begin{array}{l}\text { REACTION TIME } \\
(\mathrm{ms})\end{array}$ \\
\hline \multirow{10}{*}{ TASK } & \multirow{5}{*}{$\begin{array}{l}\text { SPEEDED } \\
\text { GO/NO GO }\end{array}$} & FAST HITS & $32.87( \pm 2.26)$ & $229.63( \pm 5.86)$ & $31.20( \pm 2.58)$ & $229.95( \pm 4.82)$ \\
\hline & & SLOW HITS & $67.13( \pm 2.26)$ & $313.71( \pm 7.16)$ & $68.80( \pm 2.58)$ & $316.14( \pm 5.96)$ \\
\hline & & ERROR & $9.11( \pm 1.11)$ & $256.69( \pm 9.99)$ & $8.60( \pm 1.11)$ & $268.78( \pm 10.61)$ \\
\hline & & $\begin{array}{l}\text { POST- } \\
\text { CORRECT }\end{array}$ & - & $-2.87( \pm 1.63)$ & - & $-1.52( \pm 1.76)$ \\
\hline & & POST-ERROR & - & $45.29( \pm 7.09)$ & - & $44.94( \pm 8.90)$ \\
\hline & \multirow{5}{*}{$\begin{array}{l}\text { SPEEDED } \\
\text { SIMON }\end{array}$} & FAST HITS & $39.07( \pm 2.60)$ & $261.53(8.24)$ & $39.28( \pm 2.09)$ & $240.80( \pm 10.17)$ \\
\hline & & SLOW HITS & $60.93( \pm 2.60)$ & $359.95( \pm 10.98)$ & $60.72( \pm 2.09)$ & $357.95( \pm 9.50)$ \\
\hline & & ERROR & $12.17( \pm 1.61)$ & $293.76( \pm 19.23)$ & $15.65( \pm 2.30)$ & $270.61( \pm 14.58)$ \\
\hline & & $\begin{array}{l}\text { POST- } \\
\text { CORRECT }\end{array}$ & - & $-3.87( \pm 1.10)$ & - & $-4.60( \pm 1.71)$ \\
\hline & & POST-ERROR & . & $56.68( \pm 7.61)$ & - & $57.50( \pm 12.03)$ \\
\hline
\end{tabular}

Table 2. Behavioral results (i.e., percentages and reaction times; means \pm 1 S.E.M), separately for each task and impact level. 


\subsubsection{Accuracy percentages and RTs.}

As intended, the reward probabilities (i.e., less frequent positive feedback vs. negative feedback) were the same for both impact conditions in the two experimental tasks. For the Go/No Go Task, participants had a larger percentage of slow hits (approximately $2 / 3)$ than fast hits $(1 / 3)$, as evident in the significant main effect of RESPONSE, $F_{(1,39)}$ $=218.960, p<0.001, \eta p^{2}=0.742$. There was no significant main effect of IMPACT, $F_{(1,39)}=0.000, p=1.000, \eta p^{2}=0.000$. Additionally, there was no significant RESPONSE x IMPACT interaction, $F_{(2,78)}=0.477, p=0.492, \eta p^{2}=0.006$, indicating a balanced asymmetry between the positive and negative feedback for the two impact conditions of the Go/No Go Task. Likewise, for the Simon Task, participants had a larger percentage of slow hits than fast hits, as revealed by the significant main effect of RESPONSE, $F_{(1,39)}=84.426, p<0.001, \eta p^{2}=0.526$. There was no significant main effect of IMPACT, $F_{(1,39)}=0.000, p=1.000, \eta p^{2}=0.000$. There was also no significant RESPONSE x IMPACT interaction, $F_{(2,78)}=0.008, p=0.929, \eta p^{2}=0.000$, suggesting a balanced asymmetry between the positive and negative feedback for the two impact conditions of the Simon Task.

Similarly, participants exhibited comparable RTs for the fast hits and slow hits between the two impact conditions. For the Go/No Go Task, the RTs for the slow hits were longer than the fast hits, as revealed by the main effect of RESPONSE, $F_{(1,39)}=$ 189.539, $p<0.001, \eta p^{2}=0.714$. There was no significant main effect of IMPACT, $F_{(1,}$ 39) $=0.449, p=0.505, \eta p^{2}=0.006$. Moreover, the difference in the RTs of the two types of responses was not modulated by the impact conditions, as illustrated by the lack of significant RESPONSE x IMPACT interaction, $F_{(2,78)}=0.003, p=0.959, \eta p^{2}=0.000$. In the same vein, for the Simon Task, the RTs for the slow hits were longer than the fast hits, as suggested by the main effect of RESPONSE, $F_{(1,39)}=118.987, p<0.001, \eta p^{2}=$ 
0.610. There was no significant main effect of IMPACT, $F_{(1,39)}=1.085, p=0.301, \eta p^{2}$ $=0.014$. Furthermore, the difference in the RTs of the two types of responses was not modulated by the impact conditions, as demonstrated by the lack of significant RESPONSE x IMPACT interaction, $F_{(2,78)}=0.700, p=0.405, \eta p^{2}=0.009$.

\subsubsection{Error percentages and RTs.}

Error commission (i.e., false alarms in the Go/No Go Task and incorrect responses in the Simon Task) of participants was also matched between the two impact conditions for both the experimental tasks. The error percentages and their corresponding RTs were comparable for the two main conditions. For the Go/No Go Task, no significant differences between the two impact conditions were observed with regard to error percentages, $t_{(38)}=0.321, p=0.750, d=0.102$, nor with regard to error RTs, $t_{(38)}=$ 0.830, $p=0.412, d=-0.262$. In the same way, for the Simon Task, no significant differences between the two impact conditions were noted with regard to the error percentages, $t_{(38)}=-1.240, p=0.223, d=-0.392$, nor with regard to error RTs, $t_{(38)}=$ $0.959, p=0.344, d=0.303$.

\subsubsection{Post-Error Slowing (PES).}

In both tasks and in both impact conditions, participants demonstrated post-error slowing (i.e., an index of behavioral adjustment following error detection that is manifested in longer RTs for correct trials after an error than correct trials not immediately after an error; Danielmeier et al., 2011; Danielmeier \& Ullsperger, 2011; King et al., 2010; Koban \& Pourtois, 2014). For the Go/No Go Task, the RTs in post-error trials were significantly longer than those in post-correct trials, as shown by the main effect of RESPONSE, $F_{(1,39)}$ 
$=66.217, p<0.001, \eta p^{2}=0.466$. There was no significant main effect of IMPACT, $F_{(1,39)}$ $=0.007, p=0.933, \eta p^{2}=0.000$. Similarly, no significant RESPONSE x IMPACT interaction was noted, $F_{(2,78)}=0.021, p=0.884, \eta p^{2}=0.000$, suggesting that post-error slowing in Go/No Go Task was matched between the two impact conditions. A similar pattern was observed in the Simon Task. The RTs in the post-error trials were significantly longer than those in the post-correct trials, as shown by the main effect of RESPONSE, $F_{(1}$, $39)=72.740, p<0.001, \eta p^{2}=0.489$. There was no significant main effect of IMPACT, $F_{(1,}$ 39) $=0.000, p=0.995, \eta p^{2}=0.000$, and no significant RESPONSE $\mathrm{x}$ IMPACT interaction, $F_{(2,78)}=0.012, p=0.914, \eta p^{2}=0.000$.

\subsection{ERP results}

\subsubsection{FRN}

\subsubsection{Mean amplitude analysis}

Mean voltage amplitude values within 250-300 ms after feedback stimulus onset from $\mathrm{Fz}$ and $\mathrm{FCz}$ electrodes for the FRN component (see Figure 4) revealed a main effect of ELECTRODE, $\left(M_{F z}=3.53 \mu \mathrm{V}, S E M=0.595 ; M_{F C z}=5.39 \mu \mathrm{V}, S E M=\right.$ $0.644), F_{(1,39)}=83.083, p<0.001, \eta p^{2}=0.681$, VALENCE, $\left(M_{\text {positive } F B}=6.35 \mu \mathrm{V}\right.$, $\left.S E M=0.610 ; M_{\text {negative } F B}=2.57 \mu \mathrm{V}, S E M=0.719\right), F_{(1,39)}=50.351, p<0.001, \eta p^{2}$ $=0.564$, and IMPACT, $F_{(1,39)}=4.759, p<0.035, \eta p^{2}=0.109$. The significant main effect of IMPACT showed that the component (N2) leading to the FRN was less negative, irrespective of valence, in the high $(M=5.08 \mu \mathrm{V}, S E M=0.597)$ compared to the low impact condition $(M=3.84 \mu \mathrm{V}, S E M=0.744)$. No significant interaction effects were observed of ELECTRODE x VALENCE, $F_{(1,39)}=2.339, p=0.134$, $\eta p^{2}=0.057$, ELECTRODE $x$ IMPACT, $F_{(1,39)}=0.129, p=0.721, \eta p^{2}=0.003$, and VALENCE x IMPACT, $F_{(1,39)}=0.347, p=0.559, \eta p^{2}=0.009$. Finally, no 
significant interaction effect of ELECTRODE x VALENCE x IMPACT was noted, $F_{(1,39)}=0.262, p=0.611, \eta p^{2}=0.007$. We carried out additional test to check for possible effect of the order of presentation of the impact conditions, taking this as a between-subjects factor (SEQUENCE). This analysis, however, did not show a significant main effect of SEQUENCE, $F_{(1,38)}=1.863, p=0.180, \eta p^{2}=0.047$. Moreover, in this analysis, the main effect of IMPACT remained significant, $F_{(1,38)}$ $=4.640, p=0.038, \eta p^{2}=0.109$.

\subsubsection{Peak-to-peak analysis}

The FRN amplitude, as quantified by the difference between the mean voltages of the P2 component (180-220 ms) and the FRN component (250-300 ms) over the Fz and FCz electrodes, revealed a main effect of ELECTRODE, $\left(M_{F z}=-4.42 \mu \mathrm{V}, S E M\right.$ $\left.=0.386 ; M_{F C z}=-4.87 \mu \mathrm{V}, S E M=0.367\right),\left(F_{(1,39)}=9.820, p=0.003, \eta p^{2}=0.201\right.$, and IMPACT, $F_{(1,39)}=8.641, p=0.005, \eta p^{2}=0.181$. The significant main effect of IMPACT showed that the component (N2) leading to the FRN was less negative, irrespective of valence, in the high $(M=-4.09 \mu \mathrm{V}, S E M=0.406)$ compared to the low impact condition $(M=-5.20 \mu \mathrm{V}, S E M=0.424)$. However, the main effect of VALENCE was only marginally significant, $\left(M_{\text {positive } F B}=-4.25 \mu \mathrm{V}, S E M=0.426\right.$; $\left.M_{\text {negative } F B}=-5.04 \mu \mathrm{V}, S E M=0.419\right), F_{(1,39)}=3.762, p=0.060, \eta p^{2}=0.088$. No significant interaction effects were observed of ELECTRODE x VALENCE, $F_{(1,39)}$ $=2.173, p=0.148, \eta p^{2}=0.053$, nor of VALENCE $\mathrm{x}$ IMPACT, $F_{(1,39)}=2.274, p=$ $0.140, \eta p^{2}=0.055$. However, significant interaction effects were obtained of ELECTRODE x IMPACT, $F_{(1,39)}=4.109, p=0.050, \eta p^{2}=0.095$, and of ELECTRODE x VALENCE x IMPACT, $F_{(1,39)}=5.505, p=0.024, \eta p^{2}=0.124$. To further examine the latter interaction, we carried out follow-up tests for each of the 
electrode sites. For the Fz channel, we observed significant main effects of VALENCE, $F_{(1,39)}=4.823, p=0.034, \eta p^{2}=0.110$, and IMPACT, $F_{(1,39)}=6.322, p$ $\left.=0.016, \eta p^{2}=0.139\right)$. Yet the VALENCE $\mathrm{x}$ IMPACT interaction was not significant, $F_{(1,39)}=0.664, p=0.420, \eta p^{2}=0.017$. For the $\mathrm{FCz}$ channel, we noted a significant main effect of IMPACT, $F_{(1,39)}=10.355, p=0.003, \eta p^{2}=0.210$, but not of VALENCE, $F_{(1,39)}=2.421, p=0.128, \eta p^{2}=0.058$. Moreover, we obtained a significant interaction effect of VALENCE x IMPACT, $F_{(1,39)}=4.127, p=0.049$, $\eta p^{2}=0.096$. Post-hoc $t$ tests in this latter channel revealed a valence-related difference (negative - positive) in the high impact condition, $t_{(39)}=-2.299, p=0.027$, $d=-0.364$, but not in the low impact condition, $t_{(39)}=-0.196, p=0.846, d=-0.031$.

\subsubsection{P3 component}

Mean voltage amplitude values within 300-400 ms after feedback stimulus onset from $\mathrm{Pz}$ and POz electrodes for the P3 component showed a main effect of ELECTRODE, $\left(M_{P z}=\right.$ $\left.9.53 \mu \mathrm{V}, S E M=0.599 ; M_{\mathrm{POz}}=7.53 \mu \mathrm{V}, S E M=0.461\right), F_{(1,39)}=58.932, p<0.001, \eta_{p}{ }^{2}=$ 0.602 and VALENCE, $\left(M_{\text {positive } F B}=9.65 \mu \mathrm{V}, S E M=0.577 ; M_{\text {negative } F B}=7.41 \mu \mathrm{V}, S E M=\right.$ $0.553), F_{(1,39)}=25.027, p<0.001, \eta_{p}^{2}=0.391$. We obtained only a marginally significant main effect of IMPACT, $F_{(1,39)}=3.936, p<0.054, \eta_{p}{ }^{2}=0.092$, with the P3 component being more positive in the high $(M=9.07 \mu \mathrm{V}, S E M=0.634)$ than the low impact condition $(M=7.98 \mu \mathrm{V}, S E M=0.537)$ irrespective of valence. A significant interaction effect was observed of ELECTRODE x VALENCE, $F_{(1,39)}=5.408, p=0.025, \eta_{p}{ }^{2}=0.122$, but not of ELECTRODE x IMPACT, $F_{(1,39)}=0.233, p=0.632, \eta_{p}{ }^{2}=0.006$, nor of VALENCE $\mathrm{x}$ IMPACT, $F_{(1,39)}=0.345, p=0.560, \eta_{p}{ }^{2}=0.009$. Finally, no significant interaction effect of ELECTRODE x VALENCE x IMPACT was detected, $F_{(1,39)}=0.586, p=0.449, \eta^{2}=$ 0.015 . 
A)
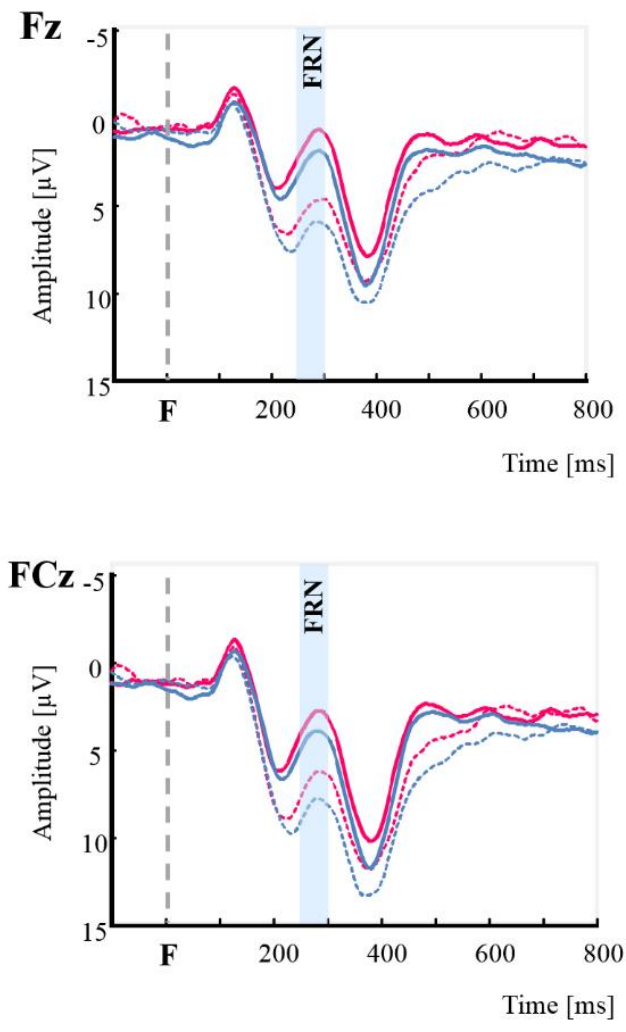

(Negative - Positive)

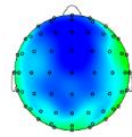

(High Impact)
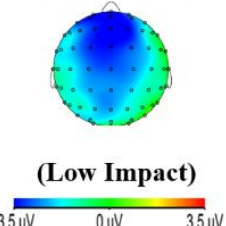

(Low - High)

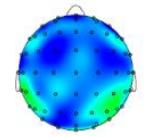

(Negative)

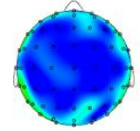

(Positive)

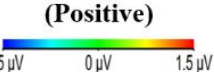

B)

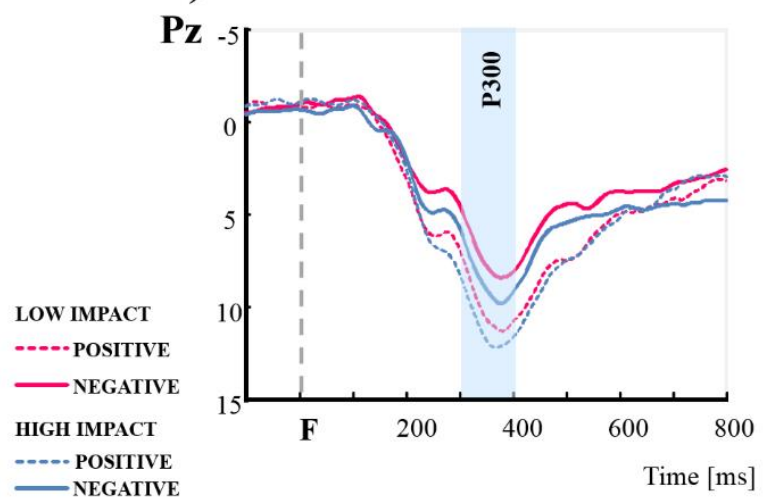

POz-

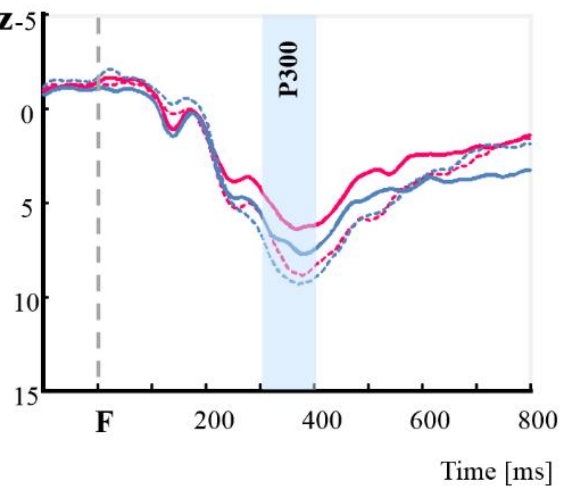

(Positive - Negative)

(High - Low)
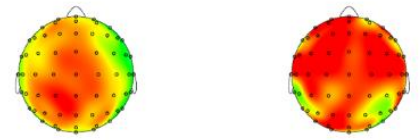

(High Impact)

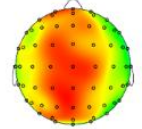

(Negative)

(Low Impact)

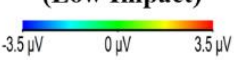

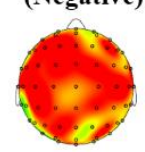

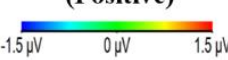

Figure 4. ERP waveforms. (A): Feedback-locked grand average waveforms for channels Fz (upper panel) and FCz (middle panel), separately for each valence and level of impact. The N2 component (giving rise to the FRN (250-300 ms post-feedback onset) when negative feedback was provided) was overall less negative in the high compared to the low impact condition, irrespective of feedback valence. The corresponding topographical scalp maps are presented (lower panel) for the FRN. (B): Feedback-locked grand average waveforms for channels Pz (upper panel) and POz (middle panel), separately for each valence and level of impact. The P3 component (300$400 \mathrm{~ms}$ post-feedback onset) was overall more positive in the high compared to the low impact condition, regardless of feedback valence. The corresponding topographical scalp maps are presented (lower panel) for the P3 component. 


\subsubsection{ERN and CRN}

The analysis showed a significant main effect of RESPONSE, $F_{(1,39)}=94.201, p<0.001$, $\eta p^{2}=0.707$, but not of ELECTRODE, $F_{(1,39)}=0.114, p=0.738, \eta p^{2}=0.003$, and IMPACT, $F_{(1,39)}=2.463, p<0.125, \eta p^{2}=0.059$. The significant main effect of RESPONSE indicated that, as expected, the ERN was more negative for errors $(M=-3.74 \mu \mathrm{V}, S E M=1.055)$ compared to hits - CRN $(M=1.66 \mu \mathrm{V}, S E M=0.830)$, irrespective of impact. Moreover, a significant interaction effect of ELECTRODE x RESPONSE was present, $F_{(1,39)}=19.541$, $p<0.001, \eta p^{2}=0.334$, but not of ELECTRODE $x$ IMPACT, $F_{(1,39)}=0.730, p=0.398, \eta p^{2}$ $=0.018$, nor of RESPONSE x IMPACT, $F_{(1,39)}=2.902, p=0.096, \eta p^{2}=0.069$. Last, the three-way ELECTRODE x RESPONSE x IMPACT interaction was not significant either, $F_{(1,39)}=0.816, p=0.372, \eta p^{2}=0.020$.

\section{Discussion}

The aim of the present study was to investigate whether changing goal impact could influence PM at the FRN (and P3) level. More specifically, we tested the prediction that performance feedback with a high goal impact would produce a larger FRN component (defined as the amplitude difference between negative and positive feedback) than performance feedback with a low goal impact, in line with previous findings (Fischer \& Ullsperger, 2013; Walentowska et al., 2016). Our main findings showed that goal impact did influence PM reliably at the FRN and P3 levels, although it did so in a valence-unspecific fashion and with opposite effects for these two successive ERP components. These results not only elucidate current understanding of higher-level modulations in PM, but they also generate interesting insights into how goal information can influence the process, which is scarcely examined in the literature. We present these empirical contributions, along with their theoretical implications, in details below. 
In this study, we ran an experiment with a crossover design in which participants completed two different cognitive control tasks (Go/No Go Task and Simon Task) that varied systematically in their degree of goal impact (low or high) via manipulation of their supposed task diagnosticity and social comparison, but were similar in terms of reward expectedness (see Table 2). Across participants, we alternated the assignment of goal impact to each task (and counterbalanced the sequence), enabling to reveal systematic effects of goal impact on PM (at the FRN and P3 levels) irrespective of possible task-specific or order-specific effects. Manipulation checks showed that while liking/disliking judgments of performance's feedback were balanced in the two goal impact conditions, participants did judge the task in the high impact condition, in which social comparison was present, to be somewhat more difficult to complete than that in the low impact condition, in which social comparison was absent. These subjective judgments, however, were not corroborated by significant differences between the two conditions in behavioral performance (i.e., accuracy, RTs, and post-error adjustments). The match in behavioral performance was an important pre-requisite for comparing the two goal impact conditions at the ERP level.

We also investigated whether the goal impact manipulation would have an influence on arousal as measured with a subjective measure (using SAM ratings) and an objective measure (via HRV). Our results clearly showed that arousal levels were balanced between the two main conditions. As expected (Hansen et al., 2003; Thayer \& Lane, 2009), the HRV (as indexed by the RMSSD) increased during task completion (suggesting a decrease in the arousal level) relative to the baseline recording, but equally so for the two impact conditions. This result suggests that the lack of reliable difference in arousal between the two conditions could not simply be imputed to a low sensitivity of the measure chosen. Likewise, SAM ratings were comparable between the two impact contexts. However, this does not imply that arousal and goal impact are necessarily decoupled in all cases. Cognitive appraisals of stimulus relating to 
its goal impact can influence affective arousal because the latter may serve as information signaling importance or urgency, hence, preparing the body for an action (Scherer, 1994; Moors, 2007; Storbeck \& Clore, 2008; Schwarz \& Clore, 2007). This may not be the case though when actions cannot be implemented.

Crucially, the ERP results showed that contrary to our prediction, we did not find a larger FRN component (discriminating negative from positive feedback) in the high than the low goal impact condition. Instead, the $\mathrm{N} 2$ component (giving rise to the FRN when negative feedback was provided) was overall less negative in the high compared to the low impact condition regardless of feedback valence, as if feedback monitoring in general was decreased in the former compared to the latter condition. By comparison, the subsequent posterior parietal P3 component was numerically larger (although marginally significant only) in the high compared to the low impact condition, again irrespective of valence. More generally, this result for the FRN suggests that our manipulation did not change reward prediction error directly. For instance, it did not make positive feedback more or less expected in the high compared to the low impact condition. Moreover, these observed goal impact modulations were confined to the externally-driven PM, and did not occur for internal PM, as revealed by our auxiliary analyses on the ERN and CRN components.

Taken all together, these new ERP results offer interesting contributions to this research domain. First, the general and valence-unspecific effects of goal impact on FRN (and P3) lend support to the assumption that goal relevance operates at a more superordinate level than valence and expectancy, which is in line with a hierarchical model of feedback-based PM (Walentowska et al., 2016). In this model, different signals, such as goal relevance, valence, and expectedness, combine and contribute to shape PM (at the FRN level), but they are situated at different places in a putative hierarchy of monitoring processes: Goal relevance is thought to operate at a more superordinate level than valence and/or expectedness (see also Badre, 2008), 
which could explain why goal relevance can change PM “directly", without necessarily altering valence or expectedness processing.

Second, although surprising at first sight, the seemingly lower feedback monitoring observed at the FRN level in the high relative to the low impact condition could be accounted for by two different (albeit not mutually exclusive) interpretations. A first interpretation is in terms of decreased mental resources. As our manipulation check showed indirectly, the thought of being socially compared in the high impact condition may have used up more mental resources during task performance compared to the low impact condition, even if this did not have a negative influence on behavioral performance. Because PM, like other cognitive processes, is subject to capacity limitations (see Badeley \& Hitch, 1974; Gilbert et al., 1988; Kahneman, 1973; Moray, 1976; Lavie, 2005; Lavie et al., 2004), it might be argued that the decreased FRN component in the high compared to the low impact condition is caused by a difference in load.

This interpretation, however, appears difficult to reconcile with the marginally significant opposite effect observed at the P3 level: The P3 had a larger amplitude in the high compared to the low impact condition, irrespective of feedback valence. This result suggests that participants presumably assigned a larger motivational significance to the feedback during PM in the high compared to the low impact condition (Bellebaum et al., 2010; Goyer et al., 2008; Gu et al., 2011; Kreussel et al., 2012; Pfabigan et al., 2011; Polezzi et al., 2010; San Martin, 2012; Toyomaki and Murohashi, 2005; Wu \& Zhou, 2009; Yeung \& Sanfey, 2004), an effect that is difficult to explain purely in terms of differences in load. This suggest a second, motivational interpretation of the observed pattern of effects. We speculate that the decreased feedback monitoring in the high impact condition reflects suppression of evaluative feedback's information as a self-protective strategy. Previous studies have shown that individuals maintain various self-protective strategies in order to maintain a positive view of themselves amidst 
innumerable threats and failures (Alicke \& Sedikides, 2011; Hoefler et al., 2015; Leary et al., 2009; Sherman \& Cohen, 2006). In our study, participants received more negative feedback than positive feedback. It is possible that participants in the high impact condition resorted to this self-protective strategy because the impact of the predominantly negative feedback was too high for their self-image or social status. We realize, however, that additional research and empirical validation are needed to corroborate more directly the link between self-protection and a reduced PM at the FRN level in this impact condition. After all, the present study is a single, preliminary effort to investigating the modulatory role of goal impact in PM. Future research could look at whether systematic changes in the type of goal under study and contextual settings (such as having more positive than negative feedback) would yield a similar FRN result.

A few limitations warrant comment. First, the goal impact manipulation may have led to the activation of different goals in the two impact conditions. So far, we assumed that this manipulation would affect the same goals-social status and/or self-esteem-but to a different degree. Yet there is a possibility that we activated slightly different goals in both conditions. For instance, the instructions in the high impact condition may have activated the proximal goal to do well on the task, as well as the distal goal to earn future academic and interpersonal success. The low impact condition, on the other hand, may have only activated the proximal goal to do well on the task. Future studies should address this limitation by dissecting more rigorously which and how many goals are activated by the chosen instructions in order to allow a better understanding of the specific contribution of each of these goals on the feedback-based PM at the level of FRN and P3 components. Additionally, because of the use of two experimental tasks used interchangeably in this study, it could be that the impact factor may have been conflated with two factors, namely, social comparison (i.e., presence or absence of it) and experimental task type (i.e., Go/No Go and Simon Tasks). Hence, the effect that we 
obtained may not reflect a pure goal impact effect. Fully orthogonalizing goal impact and experimental task type, however, is difficult in a within-subject design, because it would jeopardize the believability of the cover story (i.e. it is hard to make participants believe that the same task has a high impact at one time and a low impact at another time). Future studies with a between-subjects design might address this limitation (although such a design necessarily has other drawbacks). Other future studies may manipulate the impact for other goals than social status, to examine whether the effect that we obtained is confined to this goal or whether it extends to other goals.

Second, we did not consider possible inter-individual differences in specific dispositions or stable motivation that may have influenced the strength of the relationship observed in our study between goal impact and the FRN (and P3) component. We did not pre-screen participants along specific traits or variables. Instead, we assumed that the manipulation carried differential self-relevance to all the participants. It is possible that stable goals, such as the need for cognition (Cacioppo et al., 1996) or the need for achievement (Nicholls, 1984; Deci \& Ryan, 2000), promoted or interfered with the elected manipulation in an unclear way. Future research may need to take these more stable factors into account to examine whether they act as moderators of the effect of goal relevance on PM brain processes.

Third, we did not have a manipulation check probing for possible suspicion about the elected goal impact manipulation in relation to task diagnosticity (i.e., academic success). We maintain that the manipulation was effective, as is reflected in the observation that participants judged the low impact task to be easier than the high impact task. Still, we acknowledge that the effect was limited, and that participants' suspicion about the task diagnosticity may have contributed to this. Future studies may address this limitation by incorporating probes to detect suspicion about the deception in manipulating goal impact. 
To conclude, the present study goes beyond previous studies that showed an influence of goal relevance on PM brain processes, with a focus on the FRN (and P3) component (Walentowska et al., 2016). While previous studies focused on goal relevance of feedback in the sense of informativeness for the satisfaction status of goals, the present study focused on goal relevance of feedback in the sense of goal impact. In addition, we manipulated the degree of goal impact rather than comparing the presence or absence of goal relevance, in this way providing a more ecologically valid situation to assess the liability of PM brain processes. Our results showed that feedback monitoring at the FRN level was decreased when feedback had high compared to low impact, in the absence of differences in arousal or task involvement between both goal impact conditions. We tentatively interpret this result as reflecting a selfprotective strategy, whereby the evaluative component of PM (at the FRN level) is transiently reduced in the high compared to the low impact condition in an effort to suppress feedback information. Generally knowing less about failure of goal pursuit may have acted as a protective measure in a challenging task that has more implications to one's self-esteem and/or social status. More broadly speaking, our results add support to the notion that PM is not encapsulated and operating on the basis of motor cues only, but that it is flexibly shaped by motivational demands. This flexibility may be a pre-requisite to foster goal-adaptive behavior in everchanging social environments.

\section{Acknowledgements}

This work is supported by a research grant (number 3G024716) from the FWO (Research Foundation - Flanders) awarded to Gilles Pourtois and Agnes Moors. 


\section{References}

Aarts, K., \& Pourtois, G. (2012). Anxiety disrupts the evaluative component of performance monitoring: An ERP study. Neuropsychologia, 50(7), 1286-1296. https://doi.org/10.1016/j.neuropsychologia.2012.02.012

Aarts, K., \& Pourtois, G. (2010). Anxiety not only increases, but also alters early errormonitoring functions. Cognitive, Affective \& Behavioral Neuroscience, 10(4), 479-492. https://doi.org/10.3758/CABN.10.4.479

Alicke, M. D. \& Sedikides, C. (2011). Self-enhancement and self-protection: Historical overview and conceptual framework. In M.D. Alicke \& C. Sedikides (Eds.). Handbook of Self-enhancement and Self-protection. New York: Guilford Press.

Allain, S., Carbonnell, L., Falkenstein, M., Burle, B., \& Vidal, F. (2004). The modulation of the Ne-like wave on correct responses foreshadows errors. Neuroscience Letters, 372(12), 161-166. https://doi.org/10.1016/j.neulet.2004.09.036

Atkinson, J. W., \& Feather, N. T. (Eds.). (1966). A theory of achievement motivation. New York, NY:Wiley.

Baddeley, A.D., \& Hitch, G. (1974). Working memory. In G.H. Bower (Ed.). The Psychology of Learning and Motivation, (Vol. 8, pp. 47-89). San Diego, CA: Academic Press.

Badre, D. (2008). Cognitive control, hierarchy, and the rostro-caudal organization of the frontal lobes. Trends in Cognitive Sciences, 12(5), 193-200. https://doi.org/10.1016/j.tics.2008.02.004

Baumeister, R. F., Campbell, J. D., Krueger, J. I., \& Vohs, K. D. (2003). Does high self -esteem cause better performance, interpersonal success, happiness or healthier lifestyle? Psychological Science in the Public Interest, 4(1), 1-44. http://dx.doi.org/10.1111/15291006.01431

Bediou, B., Koban, L., Rosset, S., Pourtois, G., \& Sander, D. (2012). Delayed monitoring of accuracy errors compared to commission errors in ACC. NeuroImage, 60(4), 1925-1936. https://doi.org/10.1016/j.neuroimage.2012.02.010

Bellebaum, C., \& Daum, I. (2008). Learning-related changes in reward expectancy are reflected in the feedback-related negativity. European Journal of Neuroscience, 27(7), 1823-1835. https://doi.org/10.1111/j.1460-9568.2008.06138.x

Bellebaum, C., Polezzi, D., \& Daum, I. (2010). It is less than you expected: The feedbackrelated negativity reflects violations of reward magnitude expectations.

Neuropsychologia, 48(11), 3343-3350.

https://doi.org/10.1016/j.neuropsychologia.2010.07.023 
Berntson, G. G., \& Cacioppo, J. T. (2000). Heart Rate Variability: Stress and Psychiatric Conditions. Dynamic Electrocardiography, 57-64.

https://doi.org/10.1002/9780470987483.ch7

Bismark, A. W., Hajcak, G., Whitworth, N. M., \& Allen, J. J. B. (2013). The role of outcome expectations in the generation of the feedback-related negativity. Psychophysiology, 50(2), 125-133. https://doi.org/10.1111/j.1469-8986.2012.01490.x

Botvinick, M. M., \& Braver, T. S. (2015). Motivation and Cognitive Control: From Behavior to Neural Mechanism. Annu. Rev. Psychol, 66(1), 83-113. https://doi.org/10.1146/annurev-psych-010814-015044

Bradley, M. M. \& Lang, P. J. (1994). Measuring emotion: The self-assessment manikin and the semantic differential. Journal of Behavior Therapy and Experimental Psychiatry, 25(1), 49-59. http://dx.doi.org/10.1016/0005-7916(94)90063-9

Cacioppo, J. T., Petty, R. E., Feinstein, J. a., \& Jarvis, W. B. G. (1996). Dispositional differences in cognitive motivation: The life and times of individuals varying in need for cognition. Psychological Bulletin, 119(2), 197-253. https://doi.org/10.1037/00332909.119.2.197

Carver, C. S., \& White, T. L. (1994). Behavioral inhibition, behavioral activation, and affective responses to impending reward and punishment: The BIS/BAS scales. Journal of Personality and Social Psychology, 67, 319-333. http://dx.doi.org/10.1037/00223514.67.2.319

Cunillera, T., Fuentemilla, L., Periañez, J., Marco-Pallarès, J., Krämer, U. M., Càmara, E., ... Rodríguez-Fornells, A. (2012). Brain oscillatory activity associated with task switching and feedback processing. Cognitive, Affective \& Behavioral Neuroscience, 12(1), 16-33. https://doi.org/10.3758/s13415-011-0075-5

Deci, E. L., \& Ryan, R. M. (2000). The "What" and "Why" of Goal Pursuits: Human Needs and the Self-Determination of Behavior. Psychological Inquiry, 11(4), 227-268. https://doi.org/10.1207/S15327965PLI1104_01

Desmedt, J. E., Debecker, J., \& Manil, J. (1965). [Demonstration of a cerebral electric sign associated with the detection by the subject of a tactile sensorial stimulus. The analysis of cerebral evoked potentials derived from the scalp with the aid of numerical ordinates]. Bulletin De l'Académie Royale De Médecine De Belgique, 5, 887-936.

Dhar, M., \& Pourtois, G. (2011). Early error detection is generic, but subsequent adaption to errors is not: Evidence from ERPs. Neuropsychologia, 49, 1236-1245. http://dx.doi.org/10.1016/j.neuropsychologia.2011.01.006

Dhar, M., Wiersema, J. R., \& Pourtois, G. (2011). Cascade of neural events leading from error commission to subsequent awareness revealed using EEG source imaging. PLoS 
ONE, 6, e19578. http://dx.doi.org/10.1371/journal.pone.0019578Falkenstein M, Hohnsbein J, \& Blanke L. (1990). Effects of errors in choice reaction tasks on the ERP under focused and divided attention. In: Psychophysiological brain research (Brunia CH, Gaillard AW, Kok A, eds.), pp 192-195. Tilburg, The Netherlands: Tilburg UP.

Falkenstein, M., Hohnsbein, J., Hoormann, J., \& Blanke, L. (1990). Effects of errors in choice reaction tasks on the ERP under focused and divided attention. In: Brunia, C.H.M., Gaillard, A.W.K., Kok, A., editors. Psychophysiological Brain Research. Tilburg: Tilburg University Press, pp. 192-5.

Ferdinand, N. K., Mecklinger, A., Kray, J., \& Gehring, W. J. (2012). The processing of unexpected positive response outcomes in the mediofrontal cortex. The Journal of Neuroscience, 32(35), 12087-12092. https://doi.org/10.1523/JNEUROSCI.1410-12.2012

Fischer, A., \& Ullsperger, M. (2013). Real and fictive outcomes are processed differently but converge on a common adaptive mechanism. Neuron, 79(6), 1243-1255. https://doi.org/10.1016/j.neuron.2013.07.006

Franck, E., De Raedt, R., Barbez, C., \& Rosseel, Y. (2008). Psychometric properties of the Dutch Rosenberg Self-Esteem Scale. Psychologica Belgica, 48, 25-35. http://doi.org/10.5334/pb-48-1-25

Frank, M., J., Woroch, B. S. \& Curran, T. (2005). Error-related negativity predicts reinforcement learning and conflict biases. Neuron, 47, 495-501. http://dx.doi.org/10.1016/j.neuron.2005.06.020

Franken, I. H. A. (2002). Behavioral approach system (BAS) sensitivity predicts alcohol craving. Personality and Individual Differences, 32, 349-355. http://dx.doi.org/10.1016/S0191-8869(01)00030-7

Gehring, W. J., \& Willoughby, A. R. (2002). The medial frontal cortex and the rapid processing of monetary gains and losses. Science, 295(5563), 2279-2282. https://doi.org/10.1126/science.1066893

Gehring, W.J., Gross, B., Coles, M.G.H., Meyer, D.E., \& Donchin, E. (1993). A neural system for error detection and compensation. Psychological Science, 4, 385-390. http://journals.sagepub.com/doi/abs/10.1111/j.1467-9280.1993.tb00586.x

Gehring, W.J., Liu, Y., Orr, J.M., \& Carp, J. (2012) The error-related negativity (ERN/Ne). In: Oxford handbook of event-related potential components (Luck SJ, Kappenman E, eds), pp 231-291. New York: Oxford UP.

Gibbons, H., Schnuerch, R., \& Stahl, J. (2016). From positivity to negativity bias: Ambiguity affects the neurophysiological signatures of feedback processing. Journal of Cognitive Neuroscience, 14, 1-16. http://dx.doi.org/10.1162/jocn_a_00921 
Gilbert, D. T., Pelham, B. W., \& Krull, D. S. (1988). On Cognitive Busyness When Person Perceivers Meet Persons Perceived. Journal of Personality and Social Psychology May, 54(5), 733-740. https://doi.org/10.1037/0022-3514.54.5.733

Goyer, J. P., Woldorff, M. G., \& Huettel, S. A. (2008). Rapid electrophysiological brain responses are influenced by both valence and magnitude of monetary rewards. Journal of Cognitive Neuroscience, 20, 2058-2069. http://dx.doi.org/10.1162/jocn.2008.20134

Gratton, G., Coles, M. G., \& Donchin, E. (1983). A new method for off- line removal of ocular artifact. Electroencephalography and Clinical Neurophysiology, 55, 468-484. http://dx.doi.org/10.1016/0013-4694(83)90135-9

Gu, R., Lei, Z., Broster, L., Wu, T., Jiang, Y., \& Luo, Y. J. (2011). Beyond valence and magnitude: a flexible evaluative coding system in the brain. Neuropsychologia, 49, 38913897. http://dx.doi.org/10.1016/j.neuropsychologia.2011.10.006

Hajcak, G., Holroyd, C. B., Moser, J. S., \& Simons, R. F. (2005). Brain potentials associated with expected and unexpected good and bad outcomes. Psychophysiology, 42(2), 161170. https://doi.org/10.1111/j.1469-8986.2005.00278.x

Hajcak, G., Moser, J. S., Holroyd, C. B., \& Simons, R. F. (2006). The feedback-related negativity reflects the binary evaluation of good versus bad outcomes. Biological Psychology, 71, 148-154. http://dx.doi.org/10.1016/j.biopsycho.2005.04.001

Hajcak, G., Moser, J. S., Holroyd, C. B., \& Simons, R. F. (2007). It's worse than you thought: The feedback negativity and violations of reward prediction in gambling tasks. Psychophysiology, 44(6), 905-912. https://doi.org/10.1111/j.1469-8986.2007.00567.x

Hajihosseini, A., \& Holroyd, C. B. (2013). Frontal midline theta and N200 amplitude reflect complementary information about expectancy and outcome evaluation. Psychophysiology, 50(6), 550-562. https://doi.org/10.1111/psyp.12040

Hansen, A. L., Johnsen, B. H., \& Thayer, J. F. (2003). Vagal influence on working memory and attention. International Journal of Psychophysiology, 48(3), 263-274. https://doi.org/10.1016/S0167-8760(03)00073-4

Hoefler, A., Athenstaedt, U., Corcoran, K., Ebner, F., \& Ischebeck, A. (2015). Coping with self-threat and the evaluation of self-related traits: An fMRI study. PLoS ONE, 10(9), 116. https://doi.org/10.1371/journal.pone.0136027

Holroyd, C. B., \& Coles, M. G. H. (2002). The Neural Basis of Human Error Processing : Reinforcement Learning , Dopamine , and the Error-Related Negativity, 109(4), 679709. https://doi.org/10.1037//0033-295X.109.4.679

Holroyd, C. B., \& Yeung, N. (2012). Motivation of extended behaviors by anterior cingulate cortex, 16(2). https://doi.org/10.1016/j.tics.2011.12.008

Kahneman, D. (1973). Attention and effort. Englewood Cliffs, N.J.: Prentice-Hall. 
Kaufmann, T., Sütterlin, S., Schulz, S. M., \& Vögele, C. (2011). ARTiiFACT: a tool for heart rate artifact processing and heart rate variability analysis. Behavior Research Methods, 43(4), 1161-70. https://doi.org/10.3758/s13428-011-0107-7

Keil, A., Debener, S., Gratton, G., Junghöfer, M., Kappenman, E. S., Luck, S. J., .. Yee, C. M. (2014). Committee report: Publication guidelines and recommendations for studies using electroencephalography and magnetoencephalography. Psychophysiology, 51(1), 1-21. https://doi.org/10.1111/psyp.12147

Koban, L., \& Pourtois, G. (2014). Brain systems underlying the affective and social monitoring of actions: An integrative review. Neuroscience and Biobehavioral Reviews, 46(P1), 71-84. https://doi.org/10.1016/j.neubiorev.2014.02.014

Koban, L., Pourtois, G., Vocat, R., \& Vuilleumier, P. (2010). When your errors make me lose or win: event-related potentials to observed errors of cooperators and competitors. Social Neuroscience, 5(July 2011), 360-374. https://doi.org/10.1080/17470911003651547

Koban, L., Pourtois, G., Bediou, B., \& Vuilleumier, P. (2012). Effects of social context and predictive relevance on action outcome monitoring. Cognitive, Affective, \& Behavioral Neuroscience, 12(3), 460-478. https://doi.org/10.3758/s13415-012-0091-0

Kreussel, L., Hewig, J., Kretschmer, N., Hecht, H., Coles, M. G. H., \& Miltner, W. H. R. (2012). The influence of the magnitude, probability, and valence of potential wins and losses on the amplitude of the feedback negativity, 49, 207-219. https://doi.org/10.1111/j.1469-8986.2011.01291.x

Lane, R. D., McRae, K., Reiman, E. M., Chen, K., Ahern, G. L., \& Thayer, J. F. (2009). Neural correlates of heart rate variability during emotion. NeuroImage, 44(1), 213-222. https://doi.org/10.1016/j.neuroimage.2008.07.056

Lavie, N., Hirst, A., de Fockert, J. W., \& Viding, E. (2004). Load theory of selective attention and cognitive control. Journal of Experimental Psychology, 133(3), 339-354. https://doi.org/10.1037/0096-3445.133.3.339

Lavie, N. (2005). Distracted and confused?: Selective attention under load. Trends in Cognitive Sciences, 9(2), 75-82. https://doi.org/10.1016/j.tics.2004.12.004

Leary, M. R., Terry, M. L., Batts Allen, A., \& Tate, E. B. (2009). The concept of ego threat in social and personality psychology: is ego threat a viable scientific construct? Personality and Social Psychology Review: An Official Journal of the Society for Personality and Social Psychology, Inc, 13(3), 151-164. https://doi.org/10.1177/1088868309342595

Liebowitz, M. R. (1987). Social phobia. Modern Problems of Pharmacopsychiatry, 22, 141173.

Luck, S. J. (2014). An introduction to the event-related potential technique (2nd ed.). Cambridge, MA: MIT Press. 
Luck, S. J., \& Gaspelin, N. (2017). How to get statistically significant effects in any ERP experiment (and why you shouldn't), 54, 146-157. https://doi.org/10.1111/psyp.12639

Moors, A. (2007). Can cognitive methods be used to study the unique aspect of emotion: An appraisal theorist's answer. Cognition \& Emotion, 21(6), 1238-1269. https://doi.org/10.1080/02699930701438061

Moray, N. (1967). Where is capacity limited? A survey and model. Acta Psychologica. 27, 84-92. http://dx.doi.org/10.1016/0001-6918(67)90048-0

Miltner, W. H., Braun, C. H., \& Coles, M. G. (1997). Event-related brain potentials following incorrect feedback in a time-estimation task: evidence for a "generic" neural system for error detection. Journal of Cognitive Neuroscience, 9, 788-798. http://dx.doi.org /10.1162/jocn.1997.9.6.788

Mushtaq, F., Wilkie, R. M., Mon-Williams, M. A., \& Schaefer, A. (2016). Randomised prior feedback modulates neural signals of outcome monitoring. NeuroImage, 125, 868-879. https://doi.org/10.1016/j.neuroimage.2015.10.046

Nicholls, J. G. (1984). Achievement motivation: Conceptions of ability, subjective experience, task choice, and performance. Psychological Review, 91(3), 328-346. https://doi.org/10.1037/0033-295X.91.3.328

Nieuwenhuis, S., Holroyd, C. B., Mol, N., \& Coles, M. G. H. (2004). Reinforcement-related brain potentials from medial frontal cortex: Origins and functional significance.

Neuroscience and Biobehavioral Reviews, 28(4), 441-448.

https://doi.org/10.1016/j.neubiorev.2004.05.003

Oliveira, FT.P., Mcdonald J.J., Goodman D. (2007). Performance Monitoring in the Anterior Cingulate is Not All Error Related: Expectancy Deviation and the Representation of Action-Outcome Associations. Journal of Cognitive Neuroscience, 19, 1994-2004. http://dx.doi.org /10.1162/jocn.2007.19.12.1994

Osinsky, R., Mussel, P., \& Hewig, J. (2012). Feedback-related potentials are sensitive to sequential order of decision outcomes in a gambling task, 49, 1579-1589. https://doi.org/10.1111/j.1469-8986.2012.01473.x

Osinsky, R., Ulrich, N., Mussel, P., Feser, L., Gunawardena, A., \& Hewig, J. (2016). The feedback-related negativity reflects the combination of instantaneous and long-term values of decision outcomes. Journal of Cognitive Neuroscience, 29(3), 424-432. http://dx.doi.org /10.1162/jocn_a_01055.

Osinsky, R., Walter, H., \& Hewig, J. (2014). What is and what could have been: An ERP study on counterfactual comparisons. Psychophysiology, 51(8), 773-781. https://doi.org/10.1111/psyp.12221 
Pfabigan, D. M., Alexopoulos, J., Bauer, H., \& Sailer, U. (2011). Manipulation of feedback expectancy and valence induces negative and positive reward prediction error signals manifest in event-related brain potentials. Psychophysiology, 48(5), 656-664. https://doi.org/10.1111/j.1469-8986.2010.01136.x

Pfabigan, D. M., Zeiler, M., Lamm, C., \& Sailer, U. (2014). Blocked versus randomized presentation modes differentially modulate feedback-related negativity and P3b amplitudes. Clinical Neurophysiology, 125(4), 715-726. https://doi.org/10.1016/j.clinph.2013.09.029

Polezzi, D., Sartori, G., Rumiati, R., Vidotto, G., \& Daum, I. (2010). NeuroImage Brain correlates of risky decision-making. NeuroImage, 49(2), 1886-1894. https://doi.org/10.1016/j.neuroimage.2009.08.068

Pourtois, G. (2011). Early Error Detection Predicted by Reduced Pre-response Control Process : An ERP Topographic Mapping Study, 403-422. https://doi.org/10.1007/s10548-010-0159-5

Proudfit, G. H. (2015). The reward positivity: From basic research on reward to a biomarker for depression. Psychophysiology, 52(4), 449-459. https://doi.org/10.1111/psyp.12370

Reinhart, R. M. G., \& Woodman, G. F. (2014). Causal Control of Medial-Frontal Cortex Governs Electrophysiological and Behavioral Indices of Performance Monitoring and Learning. The Journal of Neuroscience, 34(12), 4214-4227. https://doi.org/10.1523/JNEUROSCI.5421-13.2014

Rosenberg, M. (1965). Society and the adolescent self-image. Princeton, NJ: Princeton University Press.

Rosenberg, M., Schooler, C., Schoenbach, C., \& Rosenberg, F. (1995). Global self-esteem and specific self-esteem: Different concepts, different outcomes. American Sociological Review, 60, 141-156. http://dx.doi.org/10.2307/2096350

Sambrook, T. D., \& Goslin, J. (2015). A neural reward prediction error revealed by a metaanalysis of ERPs using great grand averages. Psychological Bulletin, 141(1), 213-235. https://doi.org/10.1037/bul0000006

San Martin, R. (2012). Event-related potential studies of outcome processing and feedbackguided learning. Frontiers in Human Neuroscience, 6, 1-17.

https://doi.org/10.3389/fnhum.2012.00304

Schaefer, A., Buratto, L. G., Goto, N., \& Brotherhood, E. V. (2016). The feedback-related negativity and the $\mathrm{P} 300$ brain potential are sensitive to price expectation violations in a virtual shopping task. PLoS ONE, 11(9), 1-21. https://doi.org/10.1371/journal.pone.0163150 
Scherer, K. R. (1994). An emotion's occurrence depends on the relevance of an event to the organism's goal/need hierarchy. In P. Ekman \& R. J. Davidson (Eds.), The Nature of emotion: Fundamental questions (pp. 227-231). New York: Oxford University Press.

Sherman, D. K. \& Cohen, G. L. (2006). The psychology of self-defense: Self-affirmation theory. Advances in Experimental Social Psychology, 38, 183-242. http://dx.doi.org/10.1016/S0065-2601(06)38004-5

Schwarz, N. \& Clore, G. (2007). Feelings and phenomenal experiences. A. Kruglanski \& E. T. (Eds.), Social psychology. Handbook of basic principles, $2^{\text {nd }}$ ed (pp.385-407). New York: Guilford.

Simon, J. R., \& Rudell, A, P. (1967). Auditory S-R compatibility: The effect of an irrelevant cue on information processing. Journal of Applied Psychology, 51, 300- 304. http://dx.doi.org/10.1037/h0020586

Simmons, J. P., Nelson, L. D., \& Simonsohn, U. (2011). False-Positive Psychology. Psychological Science, 22(11), 1359-1366. https://doi.org/10.1177/0956797611417632

Stahl, J. (2010). Error detection and the use of internal and external error indicators: An investigation of the first-indicator hypothesis. International Journal of Psychophysiology, 77(1), 43-52. https://doi.org/10.1016/j.ijpsycho.2010.04.005

Storbeck, J., \& Clore, G. L. (2008). Affective Arousal as Information: How Affective Arousal Influences Judgments, Learning, and Memory. Social and Personality Psychology Compass, 2(5), 1824-1843. https://doi.org/10.1111/j.1751-9004.2008.00138.x

Sutton, S., Braren, M., Zubin, J., \& John, E. R. (1965). Evoked-potential correlates of stimulus uncertainty. Science, 150, 1187-1188. http://dx.doi.org/ 10.1126/science.150.3700.1187

Task Force of the European Society of Cardiology and the North American Society of Pacing and Electrophysiology. (1996). Heart rate variability: Standards of measurement, physiology interpretation, and clinical use. Circulation, 93, 1043-1065. https://doi.org/10.1161/01.CIR.93.5.1043

Thayer, J. F., Hansen, A. L., Saus-Rose, E., \& Johnsen, B. H. (2009). Heart rate variability, prefrontal neural function, and cognitive performance: The neurovisceral integration perspective on self-regulation, adaptation, and health. Annals of Behavioral Medicine, 37(2), 141-153. https://doi.org/10.1007/s12160-009-9101-z

Thayer, J. F., \& Lane, R. D. (2009). Claude Bernard and the heart-brain connection: Further elaboration of a model of neurovisceral integration. Neuroscience and Biobehavioral Reviews, 33(2), 81-88. https://doi.org/10.1016/j.neubiorev.2008.08.004

Toyomaki, A., \& Murohashi, H. (2005). The ERPs to feedback indicating monetary loss and gain on the game of modified "rock-paper-scissors." International Congress Series, 1278, 381-384. https://doi.org/10.1016/j.ics.2004.11.032 
Ullsperger, M., Danielmeier, C., \& Jocham, G. (2014a). Neurophysiology of performance monitoring and adaptive behavior. Physiological Reviews, 94(1), 35-79.

https://doi.org/10.1152/physrev.00041.2012

Ullsperger, M., Fischer, A. G., Nigbur, R., \& Endrass, T. (2014b). Neural mechanisms and temporal dynamics of performance monitoring. Trends in Cognitive Sciences, 18(5), 259-267. https://doi.org/10.1016/j.tics.2014.02.009

Van Vliet, I.M., Westenberg, H.G.M. (1999). Pharmacotherapy of social anxiety disorder. In: Focus on psychiatry: Social Anxiety Disorder (Westenberg, H.G.M., Den Boer, J.A., eds), Amsterdam, The Netherlands: SynThesis Publishers, pp 157-174.

Vocat, R., Pourtois, G., \& Vuilleumier, P. (2008). Unavoidable errors: A spatio-temporal analysis of time-course and neural sources of evoked potentials associated with error processing in a speeded task. Neuropsychologia, 46, 2545-2555. doi: 10.1016/j.neuropsychologia.2008. 04.006

von Borries, a K. L., Verkes, R. J., Bulten, B. H., Cools, R., \& de Bruijn, E. R. a. (2013). Feedback-related negativity codes outcome valence, but not outcome expectancy, during reversal learning. Cognitive, Affective, \& Behavioral Neuroscience, 13(4), 737-746. https://doi.org/10.3758/s13415-013-0150-1

Walentowska, W., Moors, A., Paul, K., \& Pourtois, G. (2016). Goal relevance influences performance monitoring at the level of the FRN and P3 components. Psychophysiology, 53(7), 1020-1033. https://doi.org/10.1111/psyp.12651

Walsh, M. M., \& Anderson, J. R. (2012). Learning from experience: Event-related potential correlates of reward processing, neural adaptation, and behavioral choice. Neuroscience and Biobehavioral Reviews, 36(8), 1870-1884. https://doi.org/10.1016/j.neubiorev.2012.05.008

Wu, Y., \& Zhou, X. (2009). The P300 and reward valence, magnitude, and expectancy in outcome evaluation. Brain Research, 1286, 114-122. https://doi.org/10.1016/j.brainres.2009.06.032

Yeung, N., Holroyd, C. B., \& Cohen, J. D. (2005). ERP correlates of feedback and reward processing in the presence and absence of response choice. Cerebral Cortex, 15(5), 535544. https://doi.org/10.1093/cercor/bhh153

Yeung, N., \& Sanfey, A. G. (2004). Independent coding of reward magnitude and valence in the human brain. J Neurosci, 24(28), 6258-6264. https://doi.org/10.1523/JNEUROSCI.4537-03.2004 